NBER WORKING PAPER SERIES

REGULATING BIDDER PARTICIPATION IN AUCTIONS

\author{
Vivek Bhattacharya \\ James W. Roberts \\ Andrew Sweeting \\ Working Paper 19352 \\ http://www.nber.org/papers/w19352
}
NATIONAL BUREAU OF ECONOMIC RESEARCH
1050 Massachusetts Avenue
Cambridge, MA 02138

August 2013

We are grateful to Dakshina De Silva, Timothy Dunne, Anuraddha Kankanamge, and Georgia Kosmopoulou for sharing their data with us. We would also like to acknowledge helpful discussions with and feedback from Ali Horta csu, Tim Hubbard, Jon Levin, Rob Porter, Paulo Somaini, and Steve Tadelis. Bhattacharya would like to thank the Davies Fellowship from the Duke University Department of Economics for financial support. Any errors are our own. The views expressed herein are those of the authors and do not necessarily reflect the views of the National Bureau of Economic Research.

NBER working papers are circulated for discussion and comment purposes. They have not been peerreviewed or been subject to the review by the NBER Board of Directors that accompanies official NBER publications.

(C) 2013 by Vivek Bhattacharya, James W. Roberts, and Andrew Sweeting. All rights reserved. Short sections of text, not to exceed two paragraphs, may be quoted without explicit permission provided that full credit, including $\odot$ notice, is given to the source. 
Regulating Bidder Participation in Auctions

Vivek Bhattacharya, James W. Roberts, and Andrew Sweeting

NBER Working Paper No. 19352

August 2013

JEL No. C72,D44,L20,L92

\begin{abstract} increases efficiency and reduces procurement costs significantly.

Vivek Bhattacharya

MIT Department of Economics

77 Massachusetts Avenue E19-750

Cambridge, MA 02142

bhattacv@mit.edu

James W. Roberts

Duke University

Department of Economics

213 Social Sciences Building

Durham, NC 27708

and NBER

j.roberts@duke.edu

\author{
Andrew Sweeting \\ Department of Economics \\ Tydings Hall 3266 \\ University of Maryland \\ College Park, MD 20742 \\ and NBER \\ sweeting@econ.umd.edu
}

Regulating bidder participation in auctions can potentially increase efficiency compared to standard auction formats with free entry. We show that the relative performance of two such mechanisms, a standard first-price auction with free entry and an entry rights auction, depends non-monotonically on the precision of information that bidders have about their costs prior to deciding whether to participate in a mechanism. As an empirical application, we estimate parameters from first-price auctions with free entry for bridge-building contracts in Oklahoma and Texas and predict that an entry rights auction 


\title{
Regulating Bidder Participation in Auctions
}

\author{
Vivek Bhattacharya ${ }^{1}, J_{\text {ames W. Roberts }}^{2}$, and Andrew Sweeting ${ }^{2,3}$ \\ ${ }^{1}$ Department of Economics, Massachusetts Institute of Technology \\ ${ }^{2}$ Department of Economics, Duke University and NBER \\ ${ }^{3}$ Department of Economics, University of Maryland and NBER
}

August 9, 2013

Abstract. Regulating bidder participation in auctions can potentially increase efficiency compared to standard auction formats with free entry. We show that the relative performance of two such mechanisms, a standard first-price auction with free entry and an entry rights auction, depends non-monotonically on the precision of information that bidders have about their costs prior to deciding whether to participate in a mechanism. As an empirical application, we estimate parameters from first-price auctions with free entry for bridge-building contracts in Oklahoma and Texas and predict that an entry rights auction increases efficiency and reduces procurement costs significantly.

KEYWORDS. Auctions, entry, information, procurement, selection.

JEL Codes. C72, D44, L20, L92.

\section{INTRODUCTION}

Given the importance of public procurement for many economies around the world (e.g., 12\% of GDP for OECD countries ${ }^{1}$ ) there has been a great deal of interest in, and research on, the mechanisms by which governments award projects. ${ }^{2}$ Even small improvements in a procurement mechanism's design offer the

Contact. bhattacv@mit.edu, j.roberts@duke.edu, atsweet@duke.edu. We are grateful to Dakshina De Silva, Timothy Dunne, Anuraddha Kankanamge, and Georgia Kosmopoulou for sharing their data with us. We would also like to acknowledge helpful discussions with and feedback from Ali Hortaçsu, Tim Hubbard, Jon Levin, Rob Porter, Paulo Somaini, and Steve Tadelis. Bhattacharya would like to thank the Davies Fellowship from the Duke University Department of Economics for financial support. Any errors are our own.

${ }^{1}$ See OECD (2011).

${ }^{2}$ See, for example, Bajari and Tadelis (2001) and the cites therein. 
possibility of large absolute gains in efficiency or reductions in procurement costs. An important issue that has only recently been considered ${ }^{3}$ is the fact that in many cases the firms competing for an award must engage in costly due diligence or other types of research activity in order to learn how much it will cost them to complete the job before they can submit a bid. In these cases, the procurement design may affect the set of firms making offers as well as the bids they submit.

Currently, standard first-price auctions are predominantly used for public procurement, ${ }^{4}$ and this can be partly justified on economic efficiency grounds. Existing theoretical work (Levin and Smith (1994), Gentry and Li (2012a)) has shown that when participation in auctions is costly bidders' privately optimal entry strategies into standard auction formats will be the same as would be chosen by a social planner with the same information, assuming that the planner wants to minimize the cost of completing the contract plus total entry costs. ${ }^{5}$

However, these results that show that entry decisions are efficient given the way that standard auctions work do not imply that changing the way that potential bidders enter the auction cannot raise efficiency. For example, Milgrom (2004) shows that when potential bidders have no private information about their values prior to performing due diligence (entry), efficiency is increased when the auctioneer chooses a fixed number of firms to submit bids. The intuition is quite simple: with free entry and no private information, potential bidders will mix over whether to enter (assuming moderate entry costs), resulting in a stochastic number of entrants. However, because social surplus is concave in the realized number of entrants, efficiency would be increased by fixing the number of entrants.

We compare the performance of standard auctions with free entry and "entry rights" auctions, in which potential bidders first bid, without performing due diligence, for a fixed and pre-announced number of rights to undertake due diligence and compete in a second-round auction for the contract itself. We do so under the assumption that potential bidders start with some private information (a signal) about their likely costs of completing the contract. As a result, the free-entry process will be selective in the sense that firms with lower costs will be more likely to enter a standard auction. Under this assumption it is natural for the seller, who wants to choose a fixed number of bidders, to auction off entry rights in order to select the firms that are likely to have the lowest costs. With selection, whether a standard auction with free

\footnotetext{
${ }^{3}$ See, for example, Li and Zheng (2009) or Krasnokutskaya and Seim (2011).

${ }^{4}$ See Bajari, McMillan, and Tadelis (2009).

${ }^{5}$ The typical Mankiw and Whinston (1986) result that free entry results in excess entry in a homogenous goods market does not hold because an entrant only takes business from other firms when it is socially optimal for it to do so.
} 
entry or an entry rights auction is more efficient depends on the relative importance of two factors. First, as in Milgrom's result, it is desirable to coordinate the entry decisions of different bidders, which happens in the entry rights auction but not with free entry. Second, it is desirable that the amount of entry be conditioned on the private information of the bidders, which happens with free entry since a potential bidder's entry strategy is a function of its signal, but not in the entry rights auction where the number of entrants is set by the seller in advance. ${ }^{6}$ We illustrate using an example that the trade-off between these factors depends in a non-monotonic way on the precision of the information that potential bidders have. When the information is either very precise (so that bidders know their completion costs prior to bidding) or very imprecise, the entry rights auction dominates. In the latter case, this reflects Milgrom's result. In the former case, the firm with the best signal is almost certainly the one with the lowest cost, and the entry rights auction with one entry slot can select this firm as the only entrant. However, when bidders' initial information is only moderately precise, a standard auction with free entry can dominate.

As relative performance depends on parameters, including the precision of bidders' pre-entry information, we estimate our model using data on a sample of bridge-building contracts, which were let by the Oklahoma and Texas Departments of Transportation using first-price, low-bid auctions with free entry. These types of contracts, and other forms of contracts for road construction and maintenance, are usually procured by auction. We find that entry is partially selective and that entry is costly so that, under free entry, there is substantial variation across auctions in the proportion of potential entrants that decide to enter. One of the contributions of our paper is to provide a methodology for solving and estimating first-price and entry rights auctions with endogenous and partially selective entry. We solve for bid functions and entry strategies jointly using the mathematical programming with equilibrium constraints (MPEC) approach (Su and Judd (2012)). ${ }^{7}$ We estimate our model using a simulated method of moments estimator where expectations are computed using importance sampling as suggested by Ackerberg (2009). This approach enables us to allow for rich observed and unobserved heterogeneity across auctions, unlike the one previous attempt to estimate this type of model of which we are aware (Marmer, Shneyerov, and Xu (2011)). ${ }^{8}$

\footnotetext{
${ }^{6}$ Of course, the entry decisions are conditioned on signals instead of actual costs. It is possible for a high-cost bidder to enter the auction because he received a low signal.

${ }^{7}$ Hubbard and Paarsch (2009) use this approach to solve first-price auction models with perfectly selective entry. We extend their method to allow for imperfect selection.

${ }^{8}$ Some earlier work, such as Krasnokutskaya and Seim (2011), estimates models of endogenous entry into first-price auctions with no selection. Without selection, estimation is more straightforward because the entrants into the auction can be assumed to have a random sample of values or costs.
} 
Based on our estimates, we compute that, even though entry is only partially selective, there would be substantial efficiency gains to using entry rights auctions. For example, for the representative average auction in our sample, the expected sum of contract completion costs and entry costs would be $2.14 \%$ lower in the entry rights auction format. At the same time, the entry rights auction would lower the total procurement costs of the seller by $3.57 \%$, which is much larger than the effect of the types of design changes usually considered in the literature, such as setting an optimal reserve price.

We are not aware of any previous attempts in the literature to quantify the impact of using an entry rights scheme based on estimated real-world parameters. A related theoretical literature has considered so-called "indicative bidding" schemes where potential bidders submit non-binding bids before the seller selects some of them to compete in an auction for the contract. In contrast, in an entry rights auction first-round bids are binding, and selected bidders make payments based on these bids even if they ultimately do not win the contract. Indicative schemes are often used by banks and as part of the complicated processes by which defense equipment is purchased (Hendricks and Quint (2013), Foley (2003), Welch and Fremond (1998)). Ye (2007) argued that only entry rights auctions, like the one we consider, should induce informative first-stage bids in equilibrium and so guarantee the selection of the bidders that are most likely to have the highest values in the second stage. Recently Hendricks and Quint (2013) have shown that this is not necessarily true if bidders are only allowed to choose from a discrete set of possible first-stage bids, and they provide some numerical examples comparing the performance of indicative bidding schemes and free entry auctions under this assumption. ${ }^{9}$ We consider possible explanations for why entry rights auctions are not more frequently used in practice, given our results, at the end of the paper. The paper is also related to some of our earlier work on selective entry auction models (Roberts and Sweeting (2013a), Roberts and Sweeting (2013b)). In those papers we consider selective entry into second-price auctions, where the simpler form of equilibrium bidding strategies simplifies computation and estimation relative to the first-price, low-bid auctions considered in this paper which are actually the auction format that is most widely used in practice. In Roberts and Sweeting (2013b) we compare the performance of the free entry auction with a sequential bidding process which is more efficient, and can improve the seller's revenues for a wide range of parameters, even though it allows early bidders to deter entry. Here we consider a quite different type of design change which retains the simultaneous bidding

\footnotetext{
${ }^{9}$ Kagel, Pevnitskaya, and Ye (2008) use lab experiments to compare different types of indicative bidding procedures.
} 
feature of most standardly used auctions. ${ }^{10}$

Two comments are in order about the nature of our results. First, we focus on numerical results based on estimated parameters rather than providing general theoretical results about efficiency or cost comparisons in the way that Milgrom (2004) does when there is no selection. This reflects the fact that models with selection are much less tractable, with comparisons often being parameter-dependent. We do consider how our results change when we vary the parameters of our model one at a time. A benefit of our approach is that we are able to give a clear guide to the magnitude of efficiency differences, rather than just being able to sign them. Second, we consider a specific form of entry rights auction rather than trying to consider the optimal entry rights auction. Given that the disadvantage of the entry rights auction that we consider, where the number of bidders to be selected is fixed in advance and is not conditioned on potential bidders' signals, the optimal design would likely involve making the chosen number of bidders a function of first-stage bids. We do not try to consider the optimal auction for three reasons. First, designing the optimal entry rights auction with partial selection is a very hard problem, and second, unlike the plain vanilla entry rights auction that we consider, it is likely to involve complicated procedures that neither sellers nor bidders are likely to want in practice. ${ }^{11}$ Third, our bottom-line result, given our parameters, is that the entry rights auction dominates the free entry auction. This conclusion would only be strengthened by considering the optimal entry rights auction.

The paper proceeds as follows. Section 2 presents the model of imperfectly selective entry into first-price auctions and entry rights auctions and discusses our numerical methodology for solving these models. Section 3 discusses the data. Section 4 discusses our estimation method. Section 5.1 presents and discusses the estimates of the model's parameters. The impact of employing an entry rights auction is analyzed in Section 5.2. Section 6 concludes. The Appendices contain more details of the numerical methods used to solve the first-price auctions, details of how we constructed our data sample, and Monte Carlo analysis of our estimation method.

\footnotetext{
${ }^{10}$ The percentage cost reductions from moving to an entry rights auction we estimate for the representative auction in our data are larger than the percentage revenue gains from moving to a sequential mechanism that Roberts and Sweeting (2013b) predict in their empirical setting.

${ }^{11} \mathrm{Lu}$ and $\mathrm{Ye}(2013)$ characterize the optimal entry rights auction format in a setting with no selection but where potential bidders have heterogenous entry (due-diligence) costs, showing that it can be implemented through an all-pay auction but not, more standard, uniform or discriminatory-price auctions.
} 


\section{A MODEL OF ENDOGENOUS BIDDER PARTICIPATION IN AUCTIONS}

We first introduce the general structure of costs and information in our model of procurement and then describe the standard and entry rights auctions. We conclude the section with a numerical example illustrating the intuition as to why efficiency depends on the degree of selection in the entry process and why it may be lowered by fixing the number of bidders.

A procurement agency wishes to select one of $N_{a}$ risk neutral bidders to complete a project $a$, such as constructing a bridge. The agency may require that it pay no more than a reserve price $r$ to complete the project, and if no firm submits a bid below this amount, the agency incurs a cost $c_{0}$ of procuring the work elsewhere. Potential bidders can fulfill the contract at a cost distributed $F_{C a}(\cdot)$ with compact support $[\underline{c}, \bar{c}]$ that admits a continuous density $f_{C a}(\cdot)$. We assume that bidders are ex ante symmetric and have independent private costs. To participate in the actual bidding stage of any mechanism, a potential bidder must pay an entry cost $K_{a}$. Upon paying $K_{a}$, the bidder learns its private cost for constructing the bridge, and so the entry cost is best interpreted as including the firm's cost of the research necessary to learn the cost of completing the bridge as well as participation and bidding costs. We make the assumption that firms cannot compete without paying $K_{a}$. However, prior to paying the entry cost, each firm observes a signal $S_{i}$ that is correlated with its true cost $C_{i}$ and that is not correlated with any other bidder's cost. Signals are affiliated with costs in the standard sense: the cost distribution conditional on a signal $s$ first-order stochastically dominates the cost distribution conditional on a signal $\tilde{s}<s$.

The two extreme cases of the Levin and Smith (1994) and Samuelson (1985) models (henceforth LS and $\mathrm{S}$, respectively) are of course embedded in this setup. If $S_{i}=C_{i}$, then signals are perfectly informative of costs and the setup reduces to the $\mathrm{S}$ model. When $S_{i}$ is independent of $C_{i}$, signals contain no information about costs and the setup reduces to the LS model. For many empirical settings, it seems plausible that buyers will have some, but imperfect, information about their costs prior to conducting costly research, consistent with a distribution of $S_{i}$ that is not independent of $C_{i}$ but not perfectly correlated either.

\subsection{Selective Entry into First-Price Auctions}

The first model of procurement that we introduce describes the procedure used in our data: a sealed-bid, first-price auction in which the project is awarded to the bidder that submits the lowest bid, and that bidder is paid his bid. We model this auction, which we will refer to from now on as the FPA, as having 
two stages. In the first stage, potential bidders observe their imperfectly informative signals of their costs. Based on these private signals, they take independent, simultaneous entry decisions (decide to pay $K_{a}$ ). In the second stage, all firms that paid $K_{a}$ compete in a low-bid, first-price auction with reserve price $r_{a}$. We assume that firms that do not pay $K_{a}$ cannot participate in the auction and that agents bid without knowing how many of their competitors actually entered the auction, as is done in Li and Zheng (2009). Note that it is possible that an agent who enters the auction by paying the entry cost will learn that his true cost exceeds the reserve price, in which case he will not bid.

We solve for entry decisions and bid functions that define the unique symmetric Bayesian Nash equilibrium with monotone bidding behavior. Potential bidders enter using a cutoff strategy; that is, a bidder enters if and only if he observes a signal $s_{i}<s_{a}^{*}$ for some critical value of $s_{a}^{* *}$ (Gentry and Li $(2012 \mathrm{~b}))$. Define $H_{a}(c)$ to be the probability that a given bidder either $(1)$ enters the auction and has a cost no smaller than $c$ or $(2)$ does not enter the auction; thus, $H_{a}(\cdot)$ depends on the signal cutoff value. Then, the equilibrium bid functions $\beta_{a}^{*}(\cdot)$ are given by the solutions to the optimization problem

$$
\beta_{a}^{*}(c) \equiv \underset{b}{\arg \max }(b-c)\left[H_{a}\left(\beta_{a}^{*-1}(b)\right)\right]^{N_{a}-1}
$$

The first order condition associated with this optimization problem gives the differential equation

$$
1+\beta_{a}^{*-1^{\prime}}(b)\left(b-\beta_{a}^{*-1}(b)\right)\left(N_{a}-1\right)\left[\frac{H_{a}^{\prime}\left(\beta_{a}^{*-1^{\prime}}(b)\right)}{H_{a}\left(\beta_{a}^{*-1^{\prime}}(b)\right)}\right]=0
$$

with the upper boundary condition

$$
\beta_{a}^{*}\left(r_{a}\right)=r_{a}
$$

The equilibrium critical cutoff values $s_{a}^{\prime *}$ are determined by the indifference condition that any potential bidder who receives a signal of $s_{a}^{*}$ must be indifferent between entering the auction or not paying the entry cost at all. This zero-profit condition is thus written

$$
\int_{\underline{b}_{a}}^{r_{a}}\left(b-\beta_{a}^{*-1}(b)\right) f_{C a}\left(\beta_{a}^{*-1}(b) \mid s_{a}^{*}\right)\left[H_{a}\left(\beta_{a}^{*-1}(b)\right)\right]^{N_{a}-1} d b=K_{a},
$$

where $\underline{b}_{a} \equiv \beta_{a}(\underline{c})$, and $f_{C a}(\cdot \mid s)$ is the conditional density of a bidder's costs, computed using Bayes' Rule, 
given he receives a signal $s$.

To solve for the bid functions, we adopt the MPEC approach presented by Su and Judd (2012). In a manner similar to that outlined by Hubbard and Paarsch (2009), we express the inverse bid function as a linear combination of the first $P$ Chebyshev polynomials, scaled to the interval $\left[\underline{b}_{a}, r_{a}\right]$. The choice variables in our programming problem are, therefore, $P$ Chebyshev coefficients, the signal cutoff, and the value of the low bid. We pick a fine grid $\left\{x_{j}\right\}_{i=1}^{J}$ on the interval $\left[\underline{b}_{a}, r_{a}\right]$. Then, we solve for the bid functions (more precisely, the Chebyshev coefficients) and the signal cutoff using

$$
\underset{\left\{\underline{b}_{a}, \beta_{a}^{*-1}, s_{a}^{*}\right\}}{\arg \min } \sum_{j=1}^{J} g_{a}\left(\beta_{a}^{*-1}\left(x_{j}\right)\right)^{2} \text { s.t. (3) and (4), }
$$

where $g_{a}\left(\beta_{a}^{*-1}(b)\right)$ is defined to be the left-hand side of equation (2). This nonlinear programming problem is solved using the SNOPT solver interfaced with the AMPL programming language. We give more details about the numerical methods in Appendix A.

We note that while we assume ex ante bidder symmetry, this method can also be used to solve for equilibria in auctions with multiple asymmetric potential entrants, although in this case type-symmetric equilibria may not be unique. The topic of solving for all equilibria in asymmetric first-price auction models is one of ongoing research.

\subsection{Entry Rights Auctions}

When entry is regulated, a straightforward way to identify the firms that are allowed to compete for a contract is to hold an auction. The entry rights auction, or ERA, that we consider, which is based on one proposed by Ye (2007), does just this. In the first stage of the ERA, each of the $N$ potential bidders learns his signal. Upon receiving this signal $s_{i}$, each bidder submits a first-round bid $a\left(s_{i}\right)$ for the right to participate in a second-stage auction. This bid need not be positive; if $a\left(s_{i}\right) \leq 0$, then the bidder bids for a subsidy from the procurer. The fact that $a(\cdot)$ can be negative ensures that all bidders have an incentive to participate in the first stage of the mechanism. The $n$ bidders who submit the highest first-round bids are required to participate in the second-round bidding scheme. At this time, the $(n+1)^{\text {st }}$ highest bid is revealed, and all entrants pay the procurer this bid (or receive the appropriate subsidy, if the $(n+1)^{\text {st }}$ highest bid is negative). The entrants then must spend $K$ to learn their true costs, and they subsequently 
compete in a first-price, low-bid auction with reserve $r$ that is known at the beginning of the auction. ${ }^{12}$ In the scheme we consider in this section, the first-stage bids are binding; Ye (2007) shows in his Proposition 2 that if the bids were non-binding (i.e., if the first-stage bids were merely "indicative" of the bidders' interest), no symmetric increasing equilibrium exists. In a recent paper, Hendricks and Quint (2013) show that there can be partial separation of bidder types when first-stage bids are non-binding, provided that the indicative bid space is discrete.

We are interested in a symmetric Bayesian Nash equilibrium where the first-round bidding strategy $a(\cdot)$ is monotone in the signal and the second-round bid function $\beta(\cdot)$ is monotone in the cost for every value of the revealed first-round bid. Note that in such an equilibrium, revealing the $(n+1)^{\text {st }}$ largest bid is equivalent to revealing the $(n+1)^{\text {st }}$ lowest signal; we will denote the random variable corresponding to this signal by $\bar{S}$ and refer to particular realizations of it by $\bar{s}$. Thus, entrants in the second round use Bayes' Rule to compute the distribution of the costs of any one of their opponents as $F_{C \mid S \leq \bar{s}}(\cdot)$, with density $f_{C \mid S \leq \bar{s}}(\cdot) .{ }^{13}$ Then, the second-stage bid function $\beta(\cdot ; \bar{s})$ solves the differential equation

$$
\beta^{\prime}(c ; \bar{s})=(n-1)(\beta(c ; \bar{s})-c)\left[\frac{f_{C \mid S \leq \bar{s}}(c)}{1-F_{C \mid S \leq \bar{s}}(c)}\right],
$$

with the boundary condition $\beta(r ; \bar{s})=r$. To solve for the bid function $a(\cdot)$, note that the profit of a bidder with cost $c$ who is invited to enter the auction, when the $(n+1)^{\text {st }}$ signal is $\bar{s}$, is

$$
\Pi(c ; \bar{s})=(\beta(c ; \bar{s})-c)\left[1-F_{C \mid S \leq \bar{s}}(c)\right]^{n-1} .
$$

Given that the first-round bidding takes the form of an $(n+1)^{\text {st }}$ price auction for $n$ goods, agents bid the expected profit from entering. That is,

$$
a(s)=\iint \Pi(c ; \bar{s}) d F_{C \mid S=s}(c) d F_{\bar{S} \mid \bar{S} \geq s}(\bar{s})
$$

which depends on $n$ and where $F_{C \mid S=s}(\cdot)$ is the conditional distribution of the cost given a signal of $s$, and

\footnotetext{
${ }^{12}$ To save on notation, we will drop the subscripts $a$ from the previous subsection that index auction-specific parameters.

${ }^{13}$ As Ye $(2007)$ notes, it is important to reveal the $(n+1)^{\text {st }}$ highest bid, but not winning bids, in order for bidders to enter the second stage with symmetric beliefs about their opponents. (Of course, other losing bids can be revealed as well.) If no bids were revealed before the second stage, then an entrant with signal $s_{i}$ would believe that the cost distribution of his opponents is the original cost distribution conditioned on the signal being among the lowest $n$ signals, given that $s_{i}$ is also among the $n$ lowest signals. This distribution depends on $s_{i}$.
} 
$F_{\bar{S} \mid \bar{S} \geq s}(\cdot)$ is the conditional distribution of the $(n+1)^{\text {st }}$ lowest signal $\bar{S}$ given that it is at least $s$. Note that if the minimum bid in the second round is $w$ and the $(n+1)^{\text {st }}$ highest bid in the first round is $\bar{a}$, then the cost to the procurer is $w-n \bar{a}$.

The number $n$ of firms that are allowed to enter the second stage of the ERA is a parameter that is selected by the procurer, and in most settings, the procurer would be restricted to $1 \leq n \leq N .{ }^{14}$ Note that the two extreme values $n=N$ and $n=1$ reduce to simpler mechanisms. For instance, if $n=N$, the first round of the ERA would be trivial, as all $N$ bidders would be required to pay $-K$; the setup would be equivalent to a standard FPA (in which all bidders know their true costs) with the procurer incurring an extra cost of $N \cdot K$ to subsidize all entry costs. At the other extreme, an ERA with $n=1$ is simply a one-stage auction where bidders submit bids based entirely on their signals rather than their actual costs. As long as entrants are required to pay the entry cost $K$ upon entering the second stage, this case is theoretically no different from any other. As such, throughout the remainder of this section, we will allow $n=1$ to be an admissible choice. We recognize, however, that there are practical issues with the case $n=1$. Taken literally, it corresponds to a situation where the first stage consists of the bidders bidding for the right to accept the contract at the reserve price. In such a setup, there would be no incentive for the entrant to spend $K$ to learn his own cost, and this introduces a complication into the interpretation of the entry cost in the model. As such, when we apply the model to an empirical setting in Section 5.2, we will restrict to $2 \leq n \leq N$.

\subsection{Efficiency of Bidder Entry}

We assume that the social planner in this setting would wish to minimize the winner's cost of completing the project ( or $c_{0}$ if the project is not assigned through the auction) plus the total entry costs paid by the bidders. Levin and Smith (1994) (their Proposition 6 and its corollary) and Gentry and Li (2012a) (their Proposition 4) show that in either the LS model or the affiliated signal model, symmetric equilibrium entry strategies in the FPA are socially optimal when the reserve price equals $c_{0} \cdot{ }^{15}$ However, the fact that entry strategies are socially optimal in an FPA with free entry does not imply that an alternative

\footnotetext{
${ }^{14}$ It is in principle possible to set $n=0$, of course, but doing so gives the uninteresting case in which the procurer ignores the bidders entirely and simply buys the contract from his outside option.

${ }^{15}$ In the LS model, this reserve price is optimal for the seller, although this is not true when there is selection as a higher reserve price allows the seller to extract some of the bidders' expected surplus. In our empirical setting, as we discuss below, there is no clearly specified reserve price (De Silva, Jeitschko, and Kosmopoulou (2009)).
} 
mechanism that regulates or coordinates entry, such as an ERA, cannot be more efficient. As an example, Milgrom (2004) shows that, in the case of the LS model, efficiency is improved if the seller chooses a fixed number of entrants (as happens in the ERA). ${ }^{16}$ Milgrom's result holds because in the symmetric free entry equilibrium of the LS model bidders randomize over entry, so that the realized number of entrants is stochastic, and the sum of the expected completion cost plus entry costs is convex in the realized number of entrants. ${ }^{17}$

A similar result holds in the opposite extreme of the $\mathrm{S}$ model as long as the probability that no potential bidder has a completion cost plus entry cost larger than the seller's outside option $\left(c_{0}\right)$ is small. Since bidders know their costs perfectly even before paying the entry cost, it is efficient to have at most the potential bidder with the best signal enter the second-stage auction in order to reduce entry costs. This potential bidder can be selected using an ERA where only one participant is chosen. In contrast, in an FPA with free entry no firms or more than one firm may enter. ${ }^{18}$

When there is imperfect selection, however, the FPA with free entry can outperform the ERA. This reflects the fact the amount of entry into the FPA is a function of bidders' private information about their costs (even though they make decisions independently and so the information of different potential bidders is not combined in an optimal way), which is desirable from an efficiency perspective. This is not true in the ERA since the number of entrants is fixed in advance.

To illustrate that cases with intermediate selection can be quite different, consider a simple example, in which costs are distributed lognormally with location parameter $\mu_{C}=-0.09$ and scale parameter

${ }^{16}$ This result holds as long as entry costs are not so high that, in the free entry equilibrium, no bidder enters the auction with positive probability, and not so low that all bidders enter with probability one.

${ }^{17}$ Recall that in the LS model entrants are ex ante symmetric because they have no information about their costs when entering in the FPA or when being chosen by the seller. This makes it straightforward to analyze the shape of this social inefficiency function. This is not the case in models with selection for the following reason. The efficiency of the FPA when there are $n$ realized entrants is

$$
\mathbb{E}\left[\min \left\{C_{1}, \ldots C_{n}\right\} \mid S_{i} \leq s^{\prime} \text { for all } i \in\{1,2, \ldots, n\}\right]+n K .
$$

The efficiency function for the ERA that lets in $n$ entrants is

$$
\mathbb{E}\left[\min \left\{C_{1}, \ldots C_{n}\right\} \mid\left\{S_{1}, \ldots, S_{n}\right\} \text { are the lowest } n \text { of } N \text { signals }\right]+n K .
$$

In general, these two expressions are not identical, so even if we knew that the efficiency function for the FPA were convex, this would not guarantee that fixing entry via the ERA will improve efficiency. In the LS case, of course, the two expressions are equal since $C_{i}$ is uncorrelated with $S_{i}$, and the logic from Milgrom (2004) is applicable.

${ }^{18}$ Note that the ERA will admit one entrant even if all bidders have costs larger than $c_{0}$, but as long as $r \leq c_{0}$, the FPA will not. In such a situation, the efficiency of the ERA will be lower than that of the FPA. The caveat about requiring the probability that all potential bidders have a cost plus entry cost larger than the seller's outside option to be small essentially ensures that this happens infrequently. 
$\sigma_{C}=0.2$ (implying an average cost of 0.93 with standard deviation 0.19 ) and truncated to the interval $[0,4.75]$. The cost of entry is 0.02 , there are four potential bidders, and $S_{i}=C_{i} \cdot \exp \left(\epsilon_{i}\right)$, where $\epsilon_{i}$ is distributed normally with mean 0 and standard deviation $\sigma_{\epsilon}$, with $\epsilon_{i}$ independent across bidders. We assume that $c_{0}$ and the reserve in the FPA are equal to 0.85. With this setup, $\sigma_{\epsilon}^{2}$ controls how much potential buyers know about their costs before deciding whether to enter the mechanism, and in this way it controls the amount of selection in the entry process. Holding $\sigma_{C}^{2}$ fixed, as $\sigma_{\epsilon}^{2} \rightarrow \infty$, the model will tend towards the informational assumptions of the LS model, and as $\sigma_{\epsilon}^{2} \rightarrow 0$, it tends towards the informational assumptions of the S model. Define $\alpha \equiv \sigma_{\epsilon}^{2} /\left(\sigma_{\epsilon}^{2}+\sigma_{C}^{2}\right) \cdot{ }^{19}$ This is a useful measure of the informational content of the signal and consequently the degree of selection in the entry process: a value of $\alpha$ near 1 implies that the the signal provides very little information, and the posterior distribution of the cost given the signal will be close to $F_{C}$.

[Figure 1 about here.]

Figure 1 plots both the percent difference in efficiency between the FPA and the ERA (a positive number means that the ERA is more efficient) as well as the (expected) number of entrants and the expected completion cost in each mechanism as a function of the degree of selection $(\alpha)$. Note that the completion cost is the winner's cost if the object is awarded to a bidder and is $c_{0}$ otherwise. Efficiency is the sum of the cost of completion and the entry costs paid, so that lower numbers are more efficient, and for any choice of parameters in the ERA, $n$ is set to minimize this quantity. When $\alpha$ is 0 or 1 , the ERA is more efficient, as expected based on the arguments above. However, for intermediate levels of selection, the unregulated entry process of the FPA yields a more efficient outcome.

For example, when $\alpha=0.5$, the ERA tends to award the contract to a higher cost firm, so despite the fact that it admits slightly fewer firms to the second stage than tend to enter the FPA (thus saving on entry costs paid), it is the less efficient mechanism. When $\alpha=0.6$, the reason that the ERA is less efficient than the FPA is reversed. Now the ERA awards the contract to a lower cost firm on average, but to do so, the mechanism admits more firms to the second stage than choose to enter the FPA. While these extra entrants do help to lower cost of completion in the ERA, thus improving efficiency, the entry into the ERA can be considered excessive relative to that in the FPA in the following sense: there are

${ }^{19}$ The definition of $\alpha$ comes from a property of the lognormal distribution. If the support of the lognormal distribution were not truncated, then the conditional distribution of the cost given a signal $S$ is lognormal with location parameter $\alpha \mu_{C}+(1-\alpha) \log S$ and scale parameter $\sigma_{C} \cdot \sqrt{\alpha}$. 
more firms that enter the ERA and do not win that would not enter the FPA under free entry (0.492) than vice versa (0.164). We now turn to introducing the data which we will use to estimate the above model of firm behavior to determine the relevant amount of selection in a particular empirical setting, which, by these arguments, will directly impact the potential benefits of employing an ERA there.

\section{DATA}

We consider procurement auctions for bridge construction conducted by the Oklahoma and Texas Departments of Transportation (DoTs) from March 2000 through August 2003. As described in De Silva, Dunne, Kankanamge, and Kosmopoulou (2008), data is taken from all regions of Oklahoma but only the North Texas and Panhandle regions of Texas to ensure similar geographic environments (to make projects comparable in terms of construction materials) and similar economic conditions. Interested bidders are required to purchase plans from the state (the cost of purchasing a plan is on the order of $\$ 100$ ), and the list of companies who have purchased a plan is publicly available before bidding takes place. These plans contain basic information about the project and also list an engineer's estimate.

[Table 1 about here.]

Summary statistics for the sample are presented in Table $1 .{ }^{20}$ We note that the average number of potential bidders, defined as plan holders (which is reasonable since non-plan holders cannot participate in the auction), is larger in Texas than Oklahoma, but the entry rate is roughly the same in both states. The seasonally adjusted unemployment rate, which may affect bidders' expected labor costs, is also systematically higher in Texas than in Oklahoma. The engineer's estimate for projects is higher in Texas than in Oklahoma, and the winning bids, normalized by the engineer's estimates, are also slightly higher in Texas.

Since we will apply the model presented in Section 2 to these data, we briefly discuss its main assumptions. The assumption of independent private costs is common in the literature studying highway procurement auctions; examples of papers using the IPV paradigm in highway procurement auctions include Krasnokutskaya and Seim (2011), Li and Zheng (2009) and Jofre-Benet and Pesendorfer (2003). Because our data do not include exogenous bidder characteristics that allow us to group bidders into

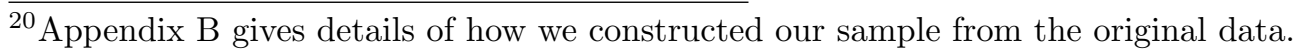


different types, we also make the common (see, for example, Li and Zheng (2009)) assumption that bidders are a priori symmetric. That only about $65 \%$ of those firms who purchase a plan actually submit a bid provides evidence of costly entry (see Krasnokutskaya and Seim (2011)). Lastly, our model assumes that there is a publicly observable reserve price in order to avoid the problem that with no reserve price, if there is some probability that other bidders do not enter, it would be optimal to submit an infinite bid. However the DoTs did not set an explicit reserve price for the auctions in our sample. We therefore impose a reserve of 1.5 times the engineer's estimate for each auction and drop (see Appendix B) the 5 out of the 471 auctions in which the declared winner bid more than this cutoff. ${ }^{21}$

\section{ESTIMATION}

In this section, we describe our estimation technique and detail the parametric specification that we take to the data. Appendix $\mathrm{C}$ provides a Monte Carlo study that analyzes the performance of the estimator.

\subsection{Model Setup and Specification}

We estimate a fully parametric version of the model from Section 2.1. We assume that $F_{C a}(\cdot)$ is a lognormal distribution with mean parameter $\mu_{C a}$ and scale parameter $\sigma_{C a}$, truncated to the interval $[0, \bar{c}] \cdot{ }^{22,23}$ Furthermore, we assume $S_{i}=C_{i} \cdot \exp \left(\epsilon_{i}\right)$, where $\epsilon_{i}$ is distributed normally with mean 0 and standard deviation $\sigma_{\epsilon a}$, with $\epsilon_{i}$ independent across bidders. As in the model in the example in Section 2.3, $\sigma_{\epsilon a}^{2}$ controls the amount of selection in the entry process and a useful measure of the informational content of the signal is $\alpha_{a}$. Given $\sigma_{C a}, \alpha_{a}$ is a strictly monotonic function of $\sigma_{\epsilon a}$. Rather than estimating $\sigma_{\epsilon a}$ directly, we choose to estimate $\alpha_{a}$ because as part of the procedure we estimate below, we have to specify bounds on the structural parameters. In the case of $\alpha_{a}, 0$ and 1 provide us with natural upper and lower bounds.

As implicit in the notation, there is auction-specific heterogeneity in the parameters $\left(\mu_{C a}, \sigma_{C a}, \alpha_{a}\right.$, and $K_{a}$ ), and we model this heterogeneity parametrically. For each auction $a$, we observe a set of covariates $X_{a}$. The specific parameters for auction $a$ are drawn from truncated normal distributions with a mean

${ }^{21} \mathrm{An}$ alternate approach (e.g., $\mathrm{Li}$ and Zheng (2009)) is to assume that the government acts as an additional bidder if only one firm enters.

${ }^{22}$ In estimation we will set $\bar{c}=4.75$ to insure that the cumulative distribution (cdf) of the lognormal distribution is very close to 1 for reasonable values of $\mu_{C}$ and $\sigma_{C}$.

${ }^{23}$ Like Krasnokutskaya and Seim (2011), our parametric assumptions are motivated by the previous literature (e.g., Porter and Zona (1993), Hong and Shum (2002), etc.). 
parameter that depends on $X_{a}$. Let $\operatorname{TRN}\left(\mu, \sigma^{2}, \underline{c}, \bar{c}\right)$ denote a normal distribution with mean $\mu$ and variance $\sigma^{2}$ truncated to the interval $[\underline{c}, \bar{c}]$. We then assume that

$$
\begin{aligned}
\mu_{C a} & \sim \operatorname{TRN}\left(X_{a} \beta_{\mu_{C}}, \omega_{\mu_{C}}^{2}, \underline{c}_{\mu_{C}}, \bar{c}_{\mu_{C}}\right) \\
\sigma_{C a} & \sim \operatorname{TRN}\left(X_{a} \beta_{\sigma_{C}}, \omega_{\sigma_{C}}^{2}, \underline{c}_{\sigma_{C}}, \bar{c}_{\sigma_{C}}\right) \\
\alpha_{a} & \sim \operatorname{TRN}\left(X_{a} \beta_{\alpha}, \omega_{\alpha}^{2}, \underline{c}_{\alpha}, \bar{c}_{\alpha}\right) \\
K_{a} & \sim \operatorname{TRN}\left(X_{a} \beta_{K}, \omega_{K}^{2}, \underline{c}_{K}, \bar{c}_{K}\right) .
\end{aligned}
$$

The truncation points are known to the econometrician. In this setting, the parameters of interest are $\Gamma \equiv\left\{\beta_{\mu_{C}}, \beta_{\sigma_{C}}, \beta_{\alpha}, \beta_{K}, \omega_{\mu_{C}}^{2}, \omega_{\sigma_{C}}^{2}, \omega_{\alpha}^{2}, \omega_{K}^{2}\right\}$. This specification allows for observed and unobserved heterogeneity across auctions in the structural parameters. Previous research (e.g. Krasnokutskaya (2011) and Bajari, Hong, and Ryan (2010)) have found significant unobserved heterogeneity in completion costs for road construction contracts.

In our setting, $X_{a}$ is a vector consisting of a constant, a dummy variable that is 1 if the state is Texas, and the county-level unemployment in the county of the project in the month of bidding. We denote the coefficients for these covariates as $\beta_{0, \times}, \beta_{1, \times}$, and $\beta_{2, \times}$, respectively, where $\times$ can be any of $\mu_{C}, \sigma_{C}$, $\alpha$, or $K$. We only allow unemployment to affect the location parameter of the cost distribution, setting $\beta_{1, \sigma_{C}}=\beta_{1, \alpha}=\beta_{1, K} \equiv 0$. The Texas dummy variable captures differences in the cost distributions of bidders, or in the procurement processes, across state lines. For estimation, we express completion costs and entry costs in terms of the engineer's estimate (so that a cost of 0.9 represents $90 \%$ of the engineer's estimate).

\subsection{Importance Sampling}

We estimate the model using a simulated method-of-moments approach with importance sampling (see Ackerberg (2009) for more on using importance sampling in estimation). The motivation behind this method is that our numerical procedure of solving auctions is relatively computationally expensive, and repeatedly solving auctions as part of a parameter optimization routine is infeasible, especially given across-auction heterogeneity and the fact that we need to solve for equilibrium bid functions in the first-price auctions. Intuitively, importance sampling allows us to estimate the parameters by first solving a large number of auctions with parameters drawn from an initial sampling distribution. Solving these 
initial auctions can be easily parallelized, and no further auctions need to be solved during the entire estimation procedure. The relevant moments from these games are then appropriately reweighted, which is computationally cheap, as the guess for the parameter of interest is changed during the estimation procedure.

To apply importance sampling to a method-of-moments approach, let $y_{a}=f\left(X_{a}, \theta_{a}\right)$ be an outcome from particular auction $t$ with observed characteristics $X_{a}$ and unobserved parameters $\theta_{a}=\left\{\mu_{C a}, \sigma_{C a}, \alpha_{a}, K_{a}\right\}$ whose distribution depends on $X_{a}$. In this setup, we will assume that the distribution of $\theta$ conditional on $X$ is characterized by a finite-dimensional parameter vector $\Gamma$, as given in equation (9). This distribution must have compact support that is known to the econometrician and does not depend on the parameters to be estimated. The sample value of the moment in $A$ data observations, conditioned on the observable covariates $X_{a}$, is then

$$
\bar{y}\left(X_{a}\right)=\frac{1}{A} \sum_{a=1}^{A} y_{a}\left(X_{a}\right) .
$$

For a particular parameter choice $\Gamma$, the expected value of the moment is

$$
\int f\left(X_{a}, \theta_{a}\right) \phi\left(\theta_{a} \mid X_{a}, \Gamma\right) d \theta
$$

where $\phi\left(\theta_{a} \mid X_{a}, \Gamma\right)$ is the pdf of $\theta_{a}$ given the observed characteristics and the parameter vector. In general, one could use a simulation-based method to compute the integral in equation (11) by drawing $S$ samples of $\theta$ from the distribution $\phi\left(\theta \mid X_{a}, \Gamma\right)$ and computing $f\left(X_{a}, \theta_{s a}\right)$ for each draw $\theta_{s a}$ so that

$$
\int f\left(X_{a}, \theta_{a}\right) \phi\left(\theta_{a} \mid X_{a}, \Gamma\right) d \theta \approx \frac{1}{S} \sum_{s=1}^{S} f\left(X_{a}, \theta_{s a}\right)
$$

In a method-of-moments estimator, $\Gamma$ is chosen to minimize the distance between the data moment in equation (10) and the simulated moments in equation (12).

Since taking new draws and solving the game for each draw for each choice of $\Gamma$ used in the optimization routine is computationally prohibitive, we can instead draw simulations from a different distribution $\psi\left(\theta_{a} \mid X_{a}\right)$ of our choosing that has the same supports and modify equation (11) as

$$
\int f\left(X_{a}, \theta_{a}\right) \frac{\phi\left(\theta_{a} \mid X_{a}, \Gamma\right)}{\psi\left(\theta_{a} \mid X_{a}\right)} \psi\left(\theta_{a} \mid X_{a}\right) d \theta
$$


Since $\psi\left(\theta_{a} \mid X_{a}\right)$ is a known density that does not depend on parameters that we seek to estimate, we can simulate this integral by taking $S$ draws of $\theta$, now from $\psi\left(\theta_{a} \mid X_{a}\right)$, and computing

$$
\frac{1}{S} \sum_{s=1}^{S} f\left(X_{a}, \theta_{a}\right) \frac{\phi\left(\theta_{s a} \mid X_{a}, \Gamma\right)}{\psi\left(\theta_{s a} \mid X_{a}\right)}
$$

Computing the pdf $\phi\left(\theta_{a} \mid X_{a}, \Gamma\right)$ is an inexpensive operation, so given a set of simulations drawn from $\psi(\cdot)$, it is computationally easy to calculate the weights on each of these draws. Equation (14) can thus be used to choose $\Gamma$ to minimize the distance between data and the simulated moments.

The moments in the data that we match are the entry distributions, winning bid distributions, and average bid distributions, conditional on the state, number of potential entrants, and the level of unemployment. When conditioning on unemployment, we divide it into two groups - auctions with high unemployment (that is, auctions with unemployment greater than the median in the specific state/time category) and those with low unemployment. ${ }^{24}$

To generate the importance sampling draws, for each observation in the data with observable covariates $X_{a}$, we take 100 draws of parameters from the distribution $\psi\left(\theta_{a} \mid X_{a}\right) \equiv \phi\left(\theta_{a} \mid X_{a}, \tilde{\Gamma}\right)$ given in Table 2 , where $\phi(\cdot)$ denotes the truncated normal model given in equation (9) with parameters $\tilde{\Gamma}$. Then for each observation in the data set with observable characteristics $X_{a}$ we randomly draw 50 solved auctions without replacement from our large pool of solved auctions with the same $X_{a}$ to form our set of simulations for the estimation procedure. The parameter estimates are chosen to match these moments in the data with the appropriately weighted moments generated from this set of simulated auctions. We estimate standard errors using a nonparametric bootstrap where we construct 250 bootstrap samples by sampling with replacement from the set of auctions in a state-number of potential bidders-level of unemployment (high or low) combination. ${ }^{25}$

[Table 2 about here.]

Appendix C reports the results of Monte Carlo experiments that indicate that this estimator can

${ }^{24}$ The "entry distribution" is the profile of the sample proportion of auctions in which a particular number of bidders enters. A particular element of the "winning bid distribution" is the sample proportion of auctions in which the winning bid falls into a pre-specified bin; we use 15 equally-sized bins that cover the interval [0, 1.5]. The "average bid distribution" tabulates the proportion of all potential bidders who submit a bid within a pre-specified bin; we use the same bins as in the winning bid distribution. Note that while the winning bid distribution sums to 1 , the average bid distribution sums to the probability of entering the bidding stage and submitting a bid.

${ }^{25}$ For each auction we draw a fresh set of 50 simulations from our large pool so that the standard errors should account for the fact that our estimates depend on the particular set of importance sampling draws used to calculate the moments. 
accurately recover the parameters even when we use a smaller number of importance sampling draws.

\subsection{Identification}

Due to data limitations and the need to account for selection, we take a fully parametric estimation approach. Assuming no unobserved across-auction heterogeneity, Gentry and Li (2012b) study nonparametric identification of a class of selective entry auction models like ours. ${ }^{26}$ They show that entry costs and the joint distribution of signals and values are exactly identified when there is sufficient exogenous variation in equilibrium entry thresholds (which in our setting could come from variation in the number of potential entrants or observable controls like the unemployment rate), and that otherwise they can be bounded. Krasnokutskaya and Seim (2011) show non-parametric identification of a first-price auction model with a non-selective entry stage when there is unobserved heterogeneity in values, although for estimation they too make parametric assumptions on model primitives. The estimation approach we use to circumvent the computational burdens associated with perhaps more standard nested fixed point methods also introduces parametric, across-auction heterogeneity in entry costs and signal noise.

\section{RESULTS}

In this section we present the estimation results and our counterfactual analysis of the impact of the DoTs switching from the current procurement format to an ERA.

\subsection{Parameter Estimates}

Table 3 gives the parameter estimates for the model with the right-hand columns showing the implied mean values, relative to cost estimates, for Oklahoma and Texas.

[Table 3 about here.]

To interpret the location and scale parameters together, we can compute the mean of the cost distribution for each auction in the data and then average across auctions in the data. This procedure shows that the mean of the cost distribution in Oklahoma is about $91.4 \%$ of the engineer's estimate (with a standard error of $0.84 \%$ ), while it is $91.9 \%$ in Texas (with a standard error of $1.89 \%$ ).

\footnotetext{
${ }^{26}$ In their conclusion they consider identification with unobserved heterogeneity that is only revealed to bidders after they enter. In contrast, we assume that they know factors that shift mean values prior to making entry decisions.
} 
Mean entry costs are 1.5\% of the engineer's estimate of the cost of the project in Oklahoma and 1.9\% in Texas. These are lower than some estimates in the literature, based on models that assume no selection (Krasnokutskaya and Seim (2011) who estimate them to be $\approx 3 \%$ and Bajari, Hong, and Ryan (2010) who estimate them to be $\approx 4.5 \%)$. This reflects the fact that we find that there is some selection in the entry process, as with selection, potential bidders may choose not to enter because they believe that they have high costs as well as the need to pay the entry cost. Our estimates are in line with guidelines from construction manuals that estimate a cost of $0.25 \%$ to $2 \%$ of the total project cost to research and prepare bids (Halpin (2005)) and that bid costs are typically about 1\% of the total bid (Park and Chapin (1992)). The difference between the parameter estimates for Oklahoma and Texas, given by the $\beta_{2}$ values, are not statistically significant. Unemployment has a negative, but statistically insignificant effect on the location parameter of the cost distribution.

The estimates in Table 3 suggest that entry is partially selective in this setting, with $\alpha$ around 0.50 in Oklahoma and 0.61 in Texas; these levels of $\alpha$ correspond to the scale parameter $\sigma_{\epsilon}$ of the error distribution being approximately $\sigma_{C}$ in Oklahoma and 1.3 times $\sigma_{C}$ in Texas. Thus, despite the fact that the amount of entry into these auctions is volatile, the fact that we find that bidders are partially informed of their costs before doing further research suggests that the government may not want to regulate participation in these auctions as it risks unnecessarily including high cost firms. We analyze this possibility in the next section.

\subsection{Regulating Bidder Entry with an Entry Rights Auction}

In this subsection, we use our parameter estimates from Section 5.1 to study the consequences of the DoTs regulating entry by selling the right to participate in these auctions. In presenting the results we assume the DoTs design the ERA to minimize their cost of procurement, and then we compare the results to those if instead they seek to maximize efficiency.

When implementing the ERAs, the auctioneer chooses to set $n$ to $n_{\mathrm{AUC}}^{*}$, the value that minimizes procurement costs. In doing so, the auctioneer balances competing effects of selling an additional entry slot. Since the auctioneer bears all entry costs in equilibrium (via the first-stage bid function $a(\cdot)),{ }^{27}$ one cost of letting an additional entrant into the auction is indeed the entry cost $K$. Additionally, by

${ }^{27}$ Conditional on $n$, the only effect that increasing the entry cost by $\Delta K$ would have is to reduce the first-round bid function to $\tilde{a}(s) \equiv a(s)-\Delta K$. 
opening an additional entry slot, the auctioneer lowers the expected profits of being admitted to the second round, and this lowers first-round indicative bids, further raising net procurement costs. However, the additional competition in the second round forces entrants to bid more aggressively, which reduces the cost of procurement.

Table 4 lists efficiencies, procurement costs, and bidder profits for auctioneer-optimal ERAs based on a number of parameters. The parameters chosen are tied to the estimates reported in Section 5.1. These estimates of course define distributions over the parameters of the auction, and Table 4 selects parameters from specific locations in the distribution. In particular, the baseline parameters presented in the first row in the table correspond to the means of the truncated normal distributions from Section 5.1, for an auction in Oklahoma with county-level unemployment equal to the mean unemployment in Oklahoma. The table also shows the results for when we vary parameters one at a time to either the $10^{\text {th }}$ or the $90^{\text {th }}$ percentiles of their respective distributions. The parameter we change is in italics in the table. We will first discuss the efficiency results and then procurement costs. For almost all parameter values considered in the comparison, the ERA results in higher efficiency (recall that this means that the cost of completion plus the sum of entry costs paid is lower) by about $2-3 \%$. The only exception is the Low $K$ case, in which the FPA is about $0.2 \%$ more efficient.

[Table 4 about here.]

We can also compute the total impact of changing from an FPA to an ERA in dollar terms by aggregating over the entire sample of projects in our data, keeping in mind that these are a small sample of the total number of road construction projects let by the DoTs during this time. For each project in the dataset, we compute the expectation of efficiency in a FPA as well as in an ERA. Across our sample, if the ERA had been used, efficiency would have been about $\$ 8.47$ million, or $2.69 \%$, higher.

To understand the differences between the two mechanisms, Table 5 breaks down the components of efficiency and procurement costs. The table presents the completion cost in the FPA as well as the entry rate, which are determined by the parameters and $s^{*}$, also reported in the table. Recall that the completion cost equals the winner's cost in cases when there is a winning bidder and equals $c_{0}$ (which is set to the reserve of 1.5) when there is no winner. In the FPA, the completion cost can be mapped to the procurement cost purely through the single bid function: these procurement costs (or winning bids) are repeated from Table 4. Table 5 also presents the completion cost in the ERA along with the bid that 
the winner submits in the second round. The last component of the procurement cost in an ERA is the first-round indicative bid paid by the entrants. The table also lists the optimal number of allowed entrants $n_{\mathrm{AUC}}^{*}$. Finally, we report the expectation of the $\left(n_{\mathrm{AUC}}^{*}+1\right)^{\text {st }}$ order statistic $\bar{S}$ of the signal distribution as a benchmark to compare to $s^{*}$ in the first-price auction. ${ }^{28}$

[Table 5 about here.]

Across the cases in Table 5, the ERA frequently allocates the project to a slightly lower cost firm and also admits fewer entrants on average (in the Base case, for instance, 3.4 firms enter the FPA on average but only 2 are selected in the ERA), thus clearly making it the more efficient mechanism. ${ }^{29}$ At times the FPA awards the project to a lower cost firm, but the ERA is still more efficient because it admits fewer firms on average. Only in the Low $K$ case do the lower total entry costs paid in the ERA not compensate enough for the fact that the FPA allocates the contract to a lower cost firm to make the ERA more efficient.

How are the rewards of the ERA's greater efficiency split between the DoTs and the firms? Returning to Table 4, we find that procurement costs could be lowered by $3-4 \%$ if the DoTs regulated bidder participation with an ERA. This drop in procurement costs is substantial, especially when compared to the impact of setting an optimal (from a procurement cost perspective) reserve price, one of the most commonly analyzed mechanism design tools. For example, in the Base case, switching to the ERA reduces procurement costs by $3.14 \%$, whereas using an optimal reserve price in an FPA only lowers procurement costs by $0.2 \% .^{30}$ Bidders, on the other hand, are hurt by the ERA, with their expected profits falling $30 \%-40 \%$.

As we did with efficiency, we can compute the total effect on procurement costs and bidder profits for projects in our data of switching to an ERA. We predict that total procurement costs would have been

${ }^{28}$ Note one important difference between $s^{*}$ and $\mathbb{E}[\bar{S}]$ : in the FPA, only $s^{*}$ is known to the entrants whereas in the ERA, the actual realization of $\bar{S}$ is revealed indirectly to the entrants through the bid they are asked to pay.

${ }^{29}$ In the FPA, there is a small but nontrivial probability that no bidder enters and the procurer has to buy the contract at a price of 1.5. This situation happens slightly less than $1 \%$ of the time in the Base case. In the ERA, however, the project is only procured from the outside option when all $n$ bidders who enter have costs larger than $r$, which never happens in our simulations. It may be reasonable to assume that when no bidder enters the FPA, the procuring agency has the option to costlessly re-run the auction, in which case we would condition all quantities in Tables 4 and 5 on at least one bidder entering the FPA. However, doing so does not change any of our results qualitatively: the completion cost of the FPA becomes slightly lower than that of the ERA, but the signs of the differences in efficiencies, procurement costs, and bidder profits remain the same, and magnitudes are not significantly different.

${ }^{30}$ We compute the optimal reserve price by searching over a grid of reserve prices with 0.01 spacing. For each reserve price we simulate procurement costs using 500,000 simulations. 
about $\$ 11.66$ million, or $3.61 \%$, lower and bidder profits would have decreased by $\$ 460,000$, or $38.6 \%$, if the ERA had been employed.

To understand these effects, we again turn to Table 5. Despite the fact that the completion cost is comparable between the FPA and the ERA, the winning bid columns for the two mechanisms show that the procurement costs in the FPA are usually significantly higher even when ignoring the revenue from the first-round bids in the ERA. In fact, for high $\sigma_{C}$ and high $\alpha$, the mean winner's second-round bid in the ERA is lower than the mean winner's bid in the FPA, even though the costs are slightly lower in the FPA. Figure 2 plots the distribution of the winner's costs as well as the procurement costs (including the first-round indicative bid in the ERA) for both mechanisms at the baseline parameters. ${ }^{31}$ The distribution of the winner's costs for the FPA and the ERA are similar in shape, with the distribution for the FPA concentrated on slightly lower values than that for the ERA. The procurement costs, however, show a marked difference in shape: the distribution for the FPA has a pronounced right tail in comparison to that of the ERA. This tail exists because the markups (defined as the ratio of the bid to the cost, less 1 ) when the winner's cost is relatively high in the FPA are larger than those in the ERA. ${ }^{32}$

[Figure 2 about here.]

The difference in markups can be seen in Figure 3, which compares the FPA bid function and the average bids from an ERA with the same parameters and $n=2 .{ }^{33}$ We also include the density functions for the cost distributions of a typical entrant into each mechanism to highlight the most empirically relevant areas of the bid functions. For this set of parameters, markups in the ERA are slightly larger than in the FPA for low-cost bidders, but they are markedly smaller for higher costs, which the majority of bidders have.

To understand these results, an important observation is that markups in the FPA are not monotonic in costs. In the FPA, both bidders with low cost and those with moderately high costs (about 0.9 to 1.1) submit bids with large markups. That weak bidders also submit high markups comes from the fact

\footnotetext{
${ }^{31}$ These parameters correspond to the Base case presented in Table 4.

${ }^{32}$ The left end of the procurement cost distribution in Figure 2(b) is also of note. Recall that in the FPA, a single bid function is used in all auctions. This bid function maps costs to the interval $[\underline{b}, r]$, and the sharp cutoff in the kernel density for the FPA corresponds to $\underline{b}$. On the other hand, the bid function in the ERA depends on the realization of $\bar{s}$, and differences in this realization lead to different minimum bids $\underline{b}$. As a result, no sharp cutoff is seen in the density for the ERA.

${ }^{33}$ Note that the average bid function in the ERA is defined by $\bar{\beta}(c) \equiv \int \beta(c ; \bar{s}) f_{\bar{S}}(\bar{s}) d \bar{s}$, where $f_{\bar{S}}(\cdot)$ is the density of the $(n+1)^{\text {st }}$ lowest signal.
} 
that the number of entrants into the auction is uncertain, and an entrant with a moderately high cost is likely to win only if no other bidders enter the auction: such entrants, therefore, submit a bid close to the reserve. For the parameters presented in this example, the average markup of the winning bid in the FPA is $9.38 \%{ }^{34}$ The ERA is much more effective at extracting profits from high-cost bidders since in an ERA, the number of actual bidders is known, and so unlike in the FPA, there is no chance that a high-cost bidder faces no competitors in the auction. This limits the incentives to offer a high markup; the average markup of the winning bidder in the ERA is only $6.76 \%$. The fact that entry is less variable in the ERA means that bidders with higher costs bid more aggressively.

[Figure 3 about here.]

We can compare these effects of switching to an ERA to those if we instead assume that the DoTs seek to maximize efficiency when choosing $n$. In doing so, they set $n=n_{\mathrm{SP}}^{*}$, which is the value that minimizes the sum of the winner's cost and the total entry costs paid. Note that the planner's problem is relatively straightforward. Given the monotonicity of $a(\cdot)$ and $\beta(\cdot ; \bar{s})$, the actual shape of the bid functions are irrelevant for the planner's problem. Indeed, the planner merely balances the cost of letting an additional entrant in with the marginal decrease in the lowest cost that comes from letting this entrant in.

Based on our estimates, the results of switching to an ERA do not greatly depend on whether the DoTs seek to minimize procurement costs or to maximize efficiency. In all ten of the eleven cases presented in Table $4, n_{\mathrm{SP}}^{*}=n_{\mathrm{AUC}}^{*}$. The only exception is when $K$ is low, in which case $n_{\mathrm{SP}}^{*}=3$. As an observation, in all the numerical experiments we have performed, we have never found a case where $n_{\mathrm{SP}}^{*}<n_{\mathrm{AUC}}^{*}$. A procurer that seeks to minimize procurement costs can exert market power by reducing the number of entry slots. Reducing the number of second-stage bidders raises first-round bids, reduces the effective entry costs the procurer pays, and does not tend to greatly raise the second-stage winning bid because the firms that do participate are those most likely to have the lowest costs of completion (and so they

\footnotetext{
${ }^{34}$ That weak bidders bid close to the reserve suggests that the reserve may play an important role in determining the expected markups of the winner and of the representative entrant. Figure 3, however, suggests that the distribution of the winner's cost - which is even farther to the left than the distribution of the representative entrant's cost - is away from the region where bids are close to the reserve. Indeed, the winner's bid is usually close to $\underline{b}$, which is still a function of $r$, of course, but does not move one-to-one with $r$. At the Base parameters, changing the reserve price over a wide range - from 1.1 to 4.75 (the maximum of the support of the cost distribution) - only changes the winner's markups from $8.3 \%$ to $10.9 \%$. The average bidder's markup does, however, change dramatically from $9.1 \%$ to $51.9 \%$. However, most of that can be attributed to a small mass of bidders bidding near these large reserve prices, which we do not see in our data. For these reasons, along with those mentioned at the end of Section 3, we consider a reserve of 1.5 to be a reasonable approximation in our setting.
} 
tend to submit lower bids). Moreover, because there is no uncertainty as to the extent of competition that bidders face, high-cost entrants no longer submit the very high markups that they would have in an unregulated entry process. As a result, we have found that for a wide variety of parameters, the social planner prefers to admit at least as many entrants to the second stage as the procurer.

Given the sizable gains of using an ERA that we estimate above, a potential puzzle is why they are not used more often for the sort of public procurement we study here. There are several possible explanations. First, it may be the case that the reduced bidder surplus in the ERA is actually harmful for the seller. In most real-world settings, the seller expects to procure many contracts over a number of years, and so will benefit when there are a large number of firms that are active in the industry and are potentially interested in bidding. The need to ensure that bidders get sufficient surplus will be even more important when many potential bidders are small firms who may face liquidity constraints, that may also be tightened by having to make binding bids in ERAs. ${ }^{35}$ A second possibility is that bidders may be risk averse. There are competing effects of risk aversion on procurement costs in the standard auction and in the ERA. While entrants will bid more aggressively, which serves to lower procurement costs, potential entrants will also be more reluctant to pay sunk entry costs to participate in an auction with an uncertain outcome, and this will lead to lower participation in both mechanisms, as well as lower indicative bids in the ERA. To evaluate whether risk aversion was a possible explanation for why ERAs are not used more widely in these auctions we computed procurement costs based on our model's estimated parameters and a variety of assumptions about the risk preferences of bidders with CARA utility. We found that for plausible levels of bidder risk aversion, the ERA was still more efficient and lowered expected procurement costs. ${ }^{36}$ Thus, risk aversion is unlikely to be the primary reason why ERAs are not more widely used. As a third possibility, Hendricks and Quint (2013) suggest that ERAs may provide sellers with perverse incentives to try to sell off contracts that, once they undertake research, bidders will find to be worthless. This seems unlikely to be a concern for state agencies that need to procure a large number of contracts and so are likely to want to maintain a reputation for probity. A fourth possibility is suggested by Kagel, Pevnitskaya, and Ye (2008), who find that in a laboratory setting that the strategic complexity of formulating offers in an ERA can lead to overbidding and bankruptcies. Whether this would hold in the field is an open

${ }^{35}$ Government agencies typically have targets for the value of contracts that should be allocated to small or minority-owned businesses which often leads them to use set-asides or bid subsidies (e.g., Krasnokutskaya and Seim (2011), Athey, Coey, and Levin (2013)).

${ }^{36}$ Previous versions of this paper contained these simulation results and they are available on request. 
question. Finally, it may of course be that some of the standard assumptions used to model procurement auctions, which we have maintained here, such as independent private values, simultaneous entry decisions and independent signals (e.g., Athey, Levin, and Seira (2011), Krasnokutskaya and Seim (2011) or Li and Zheng (2011)) are incorrect and affect the comparison of the different designs. For example, if bidder signals are correlated or entry decisions are made sequentially, entry into the FPA may be less volatile, mitigating some of the gains to using the ERA.

\section{CONCLUSION}

In procurement settings, where it is expensive for potential bidders to learn their costs of completing a project, unregulated entry can lead to volatile amounts of bidder participation. If bidders have either no information or perfect information about their costs before entering, then fixing the number of entrants, say by auctioning off entry slots as is done in an entry rights auction, can raise efficiency. Motivated by the importance of public procurement we consider whether a simple scheme that regulates entry can raise efficiency and reduce procurement costs.

When bidders' pre-entry information about their cost of completion is either very precise (so that bidders know their completion costs prior to bidding) or very imprecise, the entry rights auction dominates. However, if bidders have some pre-entry information about their costs, this need not be the case. The reason is that, unlike a standard auction into which there is free entry, the number of entrants selected by an entry rights auction is not conditioned on bidders' private pre-entry information.

Therefore, in computing the gains to regulating entry this observation highlights the importance of allowing for bidders to be imperfectly informed of their value of participation prior to entering. We do so when modeling bidder participation into bridge-building contracts in Texas and Oklahoma. We estimate that there is an intermediate amount of selection in the entry process, opening up the possibility that regulating entry may actually hurt efficiency in these auctions despite the fact that bidder participation is variable across different projects in the data. However, when we compute the gains to implementing a standard entry rights auction instead of the current free-entry first-price auction, which to our knowledge is the first quantification of the impact of using an entry rights scheme based on real-world parameters, we predict that efficiency will indeed be improved by regulating entry. We also predict that an entry rights auction will reduce procurement costs, partly by encouraging more aggressive bidding behavior 
by high cost bidders. Therefore, while in the current procurement mechanism used by the Texas and Oklahoma DoTs, firms' independent participation decisions maximize the possible efficiency afforded by such a mechanism, we predict that - despite bidders having imperfect information about their costs prior to entering - efficiency could be even greater were the DoTs to regulate entry by employing an entry rights auction.

\section{References}

Ackerberg, D. (2009): "A New Use of Importance Sampling to Reduce Computational Burden in Simulation Estimation," Quantitative Marketing and Economics, 7, 343-376.

Athey, S., D. Coey, And J. Levin (2013): "Set-Asides and Subsides in Auctions," American Economic Journal: Microeconomics, 5(1), 1-27.

Athey, S., J. Levin, And E. Seira (2011): "Comparing Open and Sealed Bid Auctions: Theory and Evidence from Timber Auctions," Quarterly Journal of Economics, 126(1), 207-257.

Bajari, P., H. Hong, And S. Ryan (2010): "Identification and Estimation of a Discrete Game of Complete Information," Econometrica, 78(5), 1529-1568.

Bajari, P., R. McMillan, and S. Tadelis (2009): "Auctions versus Negotiations in Procurement: An Empirical Analysis.," Journal of Law, Economics, and Organization, 25(2), 372 - 399.

Bajari, P., And S. Tadelis (2001): "Incentives versus Transaction Costs: A Theory of Procurement Contracts," RAND Journal of Economics, 32(3), 387-407.

De Silva, D., T. Dunne, A. Kankanamge, and G. Kosmopoulou (2008): "The Impact of Public Information on Bidding in Highway Procurement Auctions," European Economic Review, 52, 150-181.

De Silva, D., T. Jeitschko, and G. Kosmopoulou (2009): "Entry and Bidding in Common and Private Value Auctions with an Unknown Number of Rivals," Review of Industrial Organization, 35(1-2), 73-93.

Foley, J. A. (2003): "Multi-Round Auctions for Institutional Real Estate Assets: Theory and Practice," Master's thesis, MIT.

Gayle, W.-R., And J.-F. Richard (2008): "Numerical Solutions of Aysmmetric, First-Price, Independent Private Value Auctions," Computational Economics, 32, 245-278.

Gentry, M., And T. Li (2012a): “Auctions with Selective Entry," Working Paper. (2012b): "Partial Identification in Auctions with Selective Entry," Working Paper.

Halpin, D. (2005): Construction Management, 3rd Edition. Wiley.

Hendricks, K., And D. Quint (2013): "Indicative Bidding in Auctions with Costly Entry," Working Paper, University of Wisconsin.

Hong, H., And M. Shum (2002): "Increasing Competition and the Winner's Curse: Evidence from Procurement," Review of Economic Studies, 69, 871-898.

Hubbard, T., And H. Paarsch (2009): "Investigating Bid Preferences at Low-Price, Sealed-Bid Auctions with Endogenous Participation," International Journal of Industrial Organization, 27, 1-14. 
Jofre-Benet, M., And M. Pesendorfer (2003): "Estimation of a Dynamic Auction Game," Econometrica, $71(5), 1443-1489$.

Kagel, J., S. Pevnitskaya, And L. Ye (2008): "Indicative Bidding: an Experimental Analysis," Games and Economic Behavior, 62(2), 697-721.

Krasnokutskaya, E. (2011): "Identification and Estimation in Highway Procurement Auctions Under Unobserved Auction Heterogeneity," Review of Economic Studies, 78(1), 293-327.

Krasnokutskaya, E., And K. Seim (2011): "Bid Preference Programs and Participation in Highway Procurement Auctions," American Economic Review, 101(6), 2653-2686.

Levin, D., And J. Smith (1994): "Equilibrium in Auctions with Entry," American Economic Review, 84, $585-599$.

Li, T., And X. Zheng (2009): "Entry and Competition Effects in First-Price Auctions: Theory and Evidence from Procurement Auctions," Review of Economic Studies, 76, 1397-1429.

(2011): "Information Acquisition and/or Bid Preparation: A Structural Analysis of Entry and Bidding in Timber Sale Auctions," Journal of Econometrics, 168(1), 29-46.

Lu, J., AND L. YE (2013): "Efficient and Optimal Mechanisms with Private Information Acquisition Costs," Journal of Economic Theory, 148(1), 393-408.

Mankiw, N., and M. Whinston (1986): "Free Entry and Social Inefficiency," The RAND Journal of Economics, pp. $48-58$.

Marmer, V., A. Shneyerov, And P. Xu (2011): "What Model for Entry in First-Price Auctions? A Nonparametric Approach," Working Paper.

Milgrom, P. (2004): Putting Auction Theory to Work. Cambridge University Press.

OECD (ed.) (2011): Government at a Glance chap. Size of Public Procurement Market.

Park, W., And W. B. Chapin (1992): Construction Bidding: Strategic Pricing for Profit (Second Edition). Wiley.

Porter, R., And J. D. Zona (1993): "Detection of Bid Rigging in Procurement Auctions," Journal of Political Economy, 101(3), 518-538.

Roberts, J., And A. Sweeting (2013a): "Bailouts and the Preservation of Competition," Working Paper, Duke University.

(2013b): "When Should Sellers Use Auctions?," American Economic Review, 103(5), 1830-1861.

Samuelson, W. (1985): "Competitive Bidding with Entry Costs," Economic Letters, 17, 53-57.

Su, C., AND K. Judd (2012): "Constrained Optimization Approaches to Estimation of Structural Models," Econometrica, 80(5), 2213-2230.

Welch, D., And O. Fremond (1998): The Case-by-Case Approach to Privatization: Techniques and Examples. World Bank.

Ye, L. (2007): "Indicative Bidding and a Theory of Two Stage Auctions," Games and Economic Behavior, 58, $181-207$. 


\section{A. NUMERICAL METHODS TO SOLVE THE FIRST-PRICE AUCTION}

In this Appendix we detail the numerical methods used to solve for equilibrium in a first-price auction with partially selective entry. Section A.1 provides parameters, such as the degree of the polynomial expansion and the type of grid, used in the computations. It also enumerates additional constraints used in practice. Section A.2 discusses numerical checks developed on the solution to the optimization problem.

\section{A.1. Details of the Optimization Problem}

The choice of grid $\left\{x_{i}\right\}_{i=1}^{N}$ has a minor effect on the solution to the programming problem. Hubbard and Paarsch (2009) use an $N$-point Gauss-Lobatto grid on $[\underline{b}, r],{ }^{37}$ but we have found in our experiments that an evenly spaced grid is often more efficient. Throughout the paper, we use $N=500$ grid points and $P=25$ polynomials in the expansion; these choices solve the optimization problem reliably and efficiently.

Bid functions are monotonic in the cost of the bidder, so we follow Hubbard and Paarsch (2009) and impose that $\beta^{*-1}\left(x_{i}\right) \geq \beta^{*-1}\left(x_{i-1}\right)$ for $2 \leq i \leq N$. Furthermore, we impose the rationality constraint that an agent never bids less than his cost: $\beta^{*-1}\left(x_{i}\right) \leq x_{i}$ for all $i$. Finally, we replace the constraint in equation (4) by the condition that the two sides cannot differ by more than $0.1 \%$ of $K$. The integral in equation (4) is replaced by an approximation using the trapezoidal rule on the $N$-point grid defined previously.

\section{A.2. Checks on the Solution}

We implement a simple consistency check on the solved bid function $\beta^{*}$ by solving equation (1) by simulation; this check is similar to the one used by Gayle and Richard (2008), who develop a numerical method to solve for bid functions in a first-price auction in the presence of collusion. We choose a fine grid $\mathcal{B}$ of "bids." For a given cost $c$, we compute the argument of the right hand side of equation (1) for each $b \in \mathcal{B}$ by simulation, fixing $\beta^{*-1}(\cdot)$ and $s^{\prime *}$ to the values determined by the optimizer. We of course expect the optimum to be $\beta^{*}(c)$. This check amounts to computing the best response of a player conditional on all other players using the bid functions computed by the optimizer and an entry rule dictated by the computed value of $s^{\prime *}$. Figure 4 plots a sample instance of this check where $\mathcal{B}$ has step size equal to 0.003 , using the baseline parameters in the Base case in Table 4: the computed functions (solid) and the best

${ }^{37}$ A Gauss-Lobatto grid on $[-1,1]$ is defined to be the points $y_{k}=\cos [k \pi /(N-1)]$ for $k \in\{0,1, \ldots, N-1\}$. To define a grid on other intervals, we simply scale these points linearly. 
response (dashed) match each other quite closely. The major discrepancies between the bid function and the best response occur at low costs and at especially high costs. There are too few draws of bidders with low or high costs for the simulation to return meaningful results.

[Figure 4 about here.]

We can check the computed value of $s^{*}$ by computing the profits of a marginal entrant. Bayes' Rule gives the distribution of costs of an entrant who receives a signal $s^{\prime *}$. Fixing the bid functions and entry

thresholds of all agents to the computed ones and assuming that the marginal entrant will enter, we use simulation to compute the profits of the marginal entrant in the bidding stage. We expect this number to be close to the entry cost. In the baseline case again, using 500,000 simulations shows that the expected profit and the entry cost differ by less than $1 \%$.

Another method to check the value of $s^{*}$ is to use the result from Gentry and Li (2012b) that the competitive $s^{\prime}$ coincides with the social planner's choice of $s^{\prime}$. Given an $s^{\prime}$, the efficiency of an auction where bidders enter if and only if their signals are below $s^{\prime}$ can be determined by computing an integral. The value of $s^{\prime}$ that minimizes this integral is the same as the value of $s^{*}$. In the baseline case, the computed value of $s^{*}$ and the value that minimizes the integral agree to four decimal places.

\section{B. DETAILS OF SAMPLE CONSTRUCTION}

De Silva, Dunne, Kankanamge, and Kosmopoulou (2008) study auctions from January 1998 through August 2003. However, we only focus on auctions after March 2000 due to an important change in policy in Oklahoma: prior to March 2000 they did not disclose engineer's estimates to bidders. Throughout this paper, we restrict our attention to auctions with between 4 and 11 potential bidders since, as discussed in Section 4, there are very few auctions with other numbers of potential bidders. From the set of bridge construction contracts let during this period, we remove auctions from the dataset in which there was no winner or the winning bid was extremely high or low. In some auctions, companies submitted bids but the DoT did not allocate the contract to any of the bidding companies. There is no indication in the dataset to explain why the DoT did not accept any of the bids and so we drop these auctions. We remove all auctions with a winning bid less than $70 \%$ of the engineer's estimate from the dataset. Only 3 of the 161 auctions in Texas in which a winner was declared were removed due to this criterion, but 47 
of the 310 auctions in Oklahoma were removed. We justify this cutoff by noting that over one-third of the auctions that were dropped in Oklahoma (16 of the 47 auctions) were won by one of two companies, however, these companies only won 4 of the remaining 263 auctions in the sample. Furthermore, there is no noticeable observable difference between these auctions and the rest of the sample in terms of project characteristics. As described in the text, we also drop auctions with winning bids greater than $150 \%$ of the engineer's estimate.

\section{MONTE CARLO STUDIES}

To test the performance of the estimator, we conduct a set of Monte Carlo experiments using data simulated from the specification in equation (9). We draw the number of potential entrants $N$ uniformly at random from the set $\{4,6,8\}$. We set the vector of observed characteristics for auction $t$ to be $X_{a} \equiv\left(1, x_{a}\right)$ where $x_{a}$ is drawn uniformly at random from the set $\{0,0.2,0.4,0.6,0.8,1\}$. The remaining parameters in the specification are taken to be $\beta_{\mu_{C}}=(0,0.65)^{\prime}, \beta_{\sigma_{C}}=(0.05,0)^{\prime}, \beta_{\alpha}=(0.5,0)^{\prime}, \beta_{K}=(0.04,0.05)^{\prime}$, $\omega_{\mu_{C}}=0.05, \omega_{\sigma_{C}}=0.02, \omega_{\alpha}=0.05$, and $\omega_{K}=0.015$. We denote this "true" parameter vector, consisting of all $\beta$ and $\omega$ parameters, as $\Gamma_{0}$ throughout this section. The truncation points are taken to be $\underline{c}_{\mu_{C}}=-0.4$, $\bar{c}_{\mu_{C}}=1, \underline{c}_{\sigma_{C}}=0.005, \bar{c}_{\sigma_{C}}=0.995, \underline{c}_{\alpha}=0.1, \bar{c}_{\alpha}=0.9, \underline{c}_{K}=0.0001$, and $\bar{c}_{K}=0.16$. These truncation points are chosen to ensure that the solver can determine a correct solution for the auction with any set of parameters drawn from the above distribution. The reserve price is set of 4.75 , a value that is beyond the $99 \%$ quantile for most $\left(\mu_{C}, \sigma_{C}\right)$ pairs drawn from the specification. As a result, the reserve is relatively non-binding. For each set of parameters drawn from this distribution, we solve for the equilibrium bid functions and entry decisions and generate a single simulation. We assume that the observable data are the potential number of entrants, $x_{a}$, the entry decision of each potential entrant, and the bids of all agents who chose to enter, as is the case in our empirical application.

We separate the data by groups consisting of the same $N$ and $x_{a}$ values. For each such group of auctions, we calculate

1. the number of potential entrants who enter but do not submit a bid (since their valuations are above the reserve),

2. the distribution of the number of entrants, 
3. the distribution of the minimum bid, and

4. the distribution of all bids.

The distribution of the number of entrants is essentially an $(N+1)$-element vector where the $k^{\text {th }}$ element gives the proportion of auctions in which $k-1$ bidders entered. The distribution of the bids are similarly discretized as well. In particular, for the distribution of the minimum bid, we divide the interval $[0.4,2.65]$ into bins of width 0.075 . For the distribution of all bids, we subdivide the interval $[0.4,4.2]$ into bins of width 0.1 .

We perform two experiments. First, we compute the infeasible estimator by using the true distribution as the importance sampling density. Using notation from Section $4.2, \psi\left(\theta_{a} \mid X_{a}\right) \equiv \phi\left(\theta_{a} \mid X_{a}, \Gamma_{0}\right)$. We begin by drawing 25,000 values of $N$ and $x_{a}$. These variables are drawn uniformly from the discrete distributions specified above. For each of these draws, a single $\theta_{a}$ is drawn from the distribution specified by $\Gamma_{0}$; the draw of $\theta_{a}$ of course depends on the draw of $x_{a}$. Finally, for each draw of $\theta_{a}$, we simulate a single auction and record the information detailed above.

The Monte Carlo experiment proceeds by first selecting 500 of these auctions as data. We then choose 2,500 other auctions as importance sampling simulations for importance sampling, which corresponds to using 5 importance sampling simulations per data auction. The simulated moments are calculated for a particular choice of $\Gamma$ by appropriately weighting the moments from this simulation set. The parameter $\Gamma$ is then chosen to minimize the distance between the moments for the data and the moments of the importance sampling simulation, with the identity as the weighting matrix. ${ }^{38}$ This procedure is repeated 100 times by reselecting both the data and simulations and subsequently recalculating $\Gamma$. Column (A) in Table 6 shows both the means and the standard deviations over the 100 runs. When sampling from the true distribution, all parameters are recovered accurately.

[Table 6 about here.]

We also run a second experiment where the importance sampling simulations are drawn from a wider distribution $\Gamma^{\prime}$ where $\omega_{\mu_{C}}=0.1, \omega_{\sigma_{C}}=0.04, \omega_{\alpha}=0.1$, and $\omega_{K}=0.03$ and all other parameters are the same as in $\Gamma_{0}$. The data runs are still drawn from the distribution $\Gamma_{0}$ specified earlier. We generate

${ }^{38}$ We have found that the objective function is smooth and we conduct this minimization procedure using Matlab's built-in optimization routine lsqnonlin. The initial guess is set to the true value, but we have found through experimentation that initial guesses on the same order of magnitude return optimal points that are numerically identical. 
75,000 simulations from this distribution. For each Monte Carlo run, we choose 500 auctions from the true distribution as the data runs and 2,500 auctions from the wider distribution as importance sampling simulations. The results over 100 Monte Carlo runs are given in column (B). We repeat the Monte Carlo runs using 15,000 simulation auctions for each run (corresponding to 30 simulations per auction), and the results over 100 runs are given in column (C). Even with 5 importance sampling simulations per auction, the Monte Carlos effectively recover the true parameters for the constant and the value of the dependence on $x_{a}$. Estimates of the standard deviations $\omega$ are biased slightly when using 5 simulations per auction, but they become closer to the true values when using 30 simulations per auction. 


\section{FIGURES}
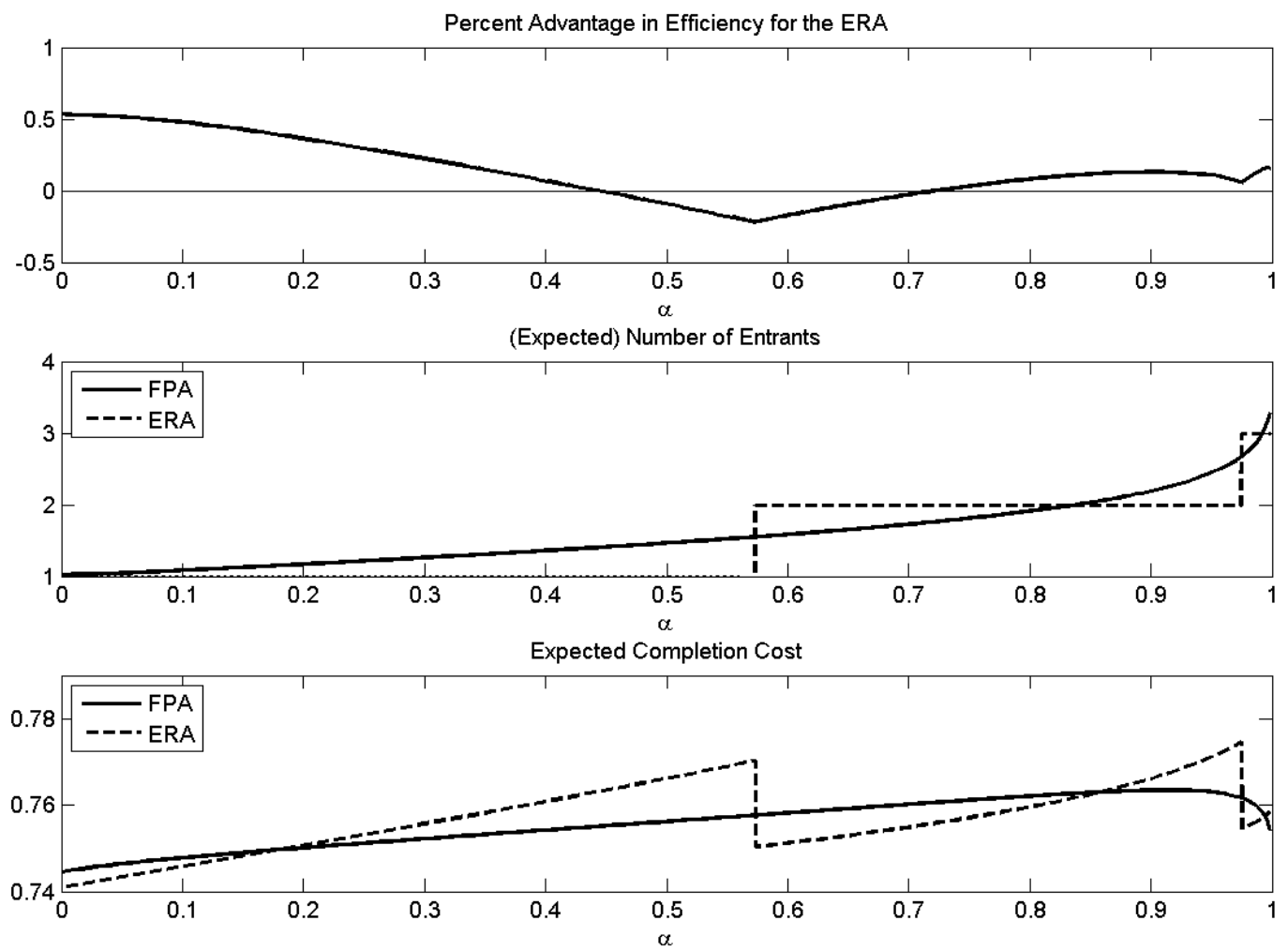

Figure 1: Comparing efficiency, amount of entry and the expected completion cost in the FPA and in the ERA when $F_{C}(c) \sim L N(-0.09,0.2), K=0.02, N=4, r=c_{0}=0.85$, and $S_{i}=C_{i} \cdot \exp \left(\epsilon_{i}\right)$. 


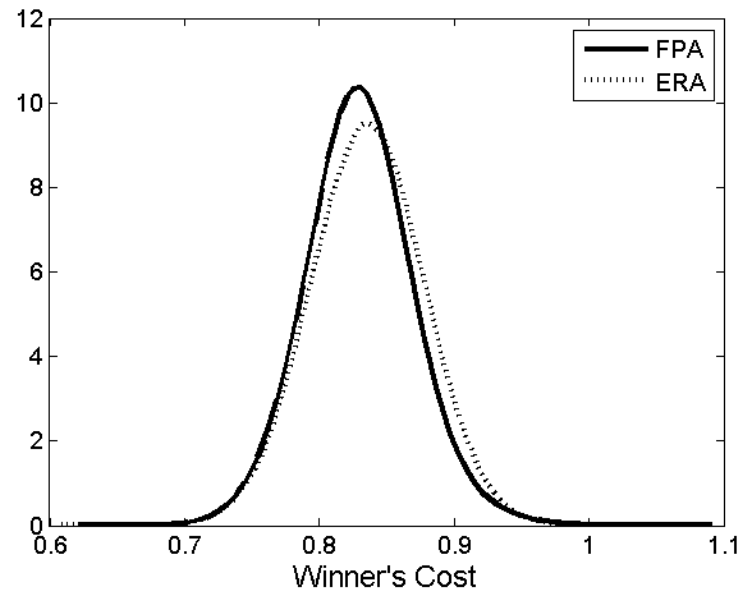

(a) Winner's cost

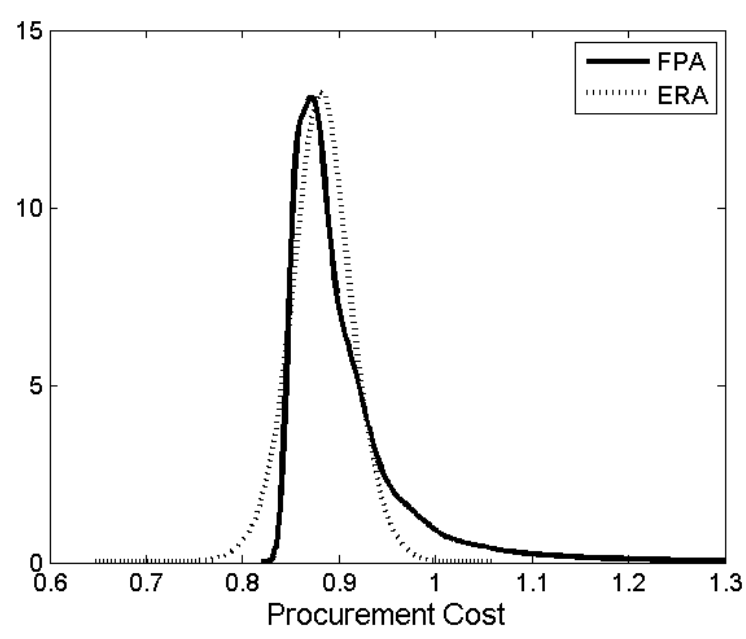

(b) Procurement Cost

Figure 2: Kernel densities for (a) the winner's cost and (b) total procurement costs to the seller in both the FPA and the ERA, using the baseline parameters. The costs to the procurer in the ERA include the revenue from the first-stage indicative bids. Densities are plotted based on 500,000 simulations, and simulations of the FPA in which no bidders entered are ignored.

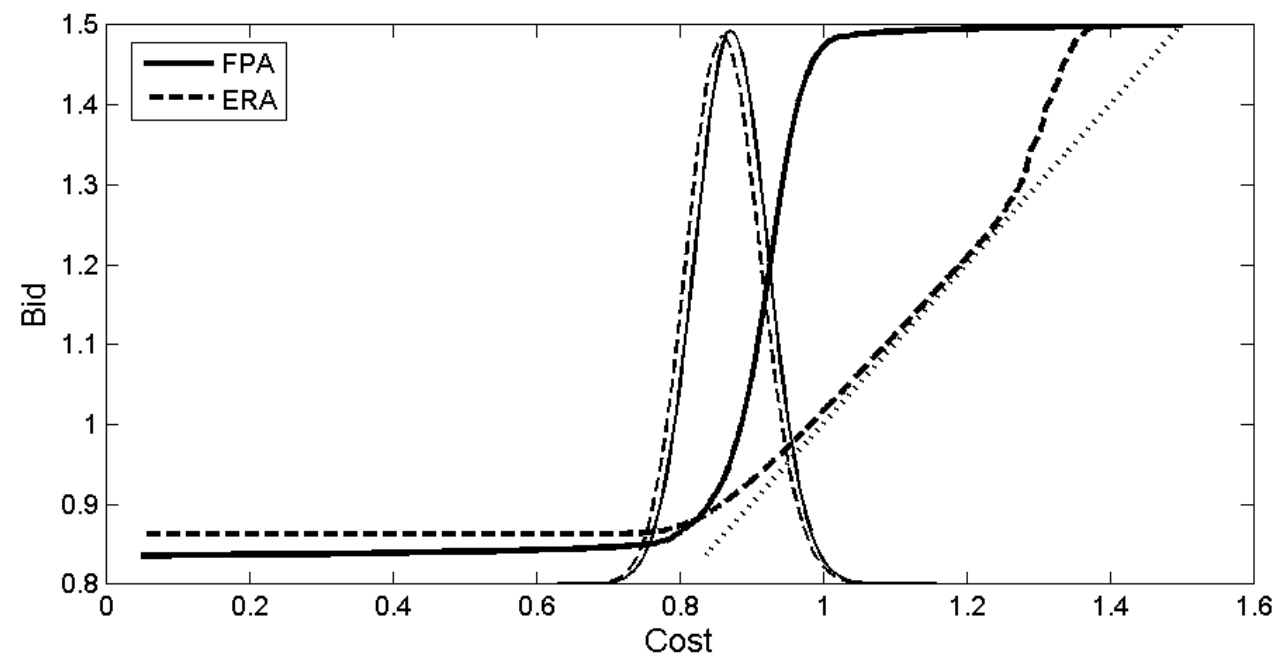

Figure 3: Comparing the bid function for a FPA (solid line) with the average bid function in an ERA with $n=2$ (dashed line). The parameters are $\mu_{C}=-0.0963, \sigma_{C}=0.0705, \alpha=0.4979, K=0.0147, N=7$, and a reserve price $r=1.5$. The dotted line is the $45^{\circ}$ line, for comparison. Densities for the cost of a typical entrant into both the FPA and the ERA are also plotted. 


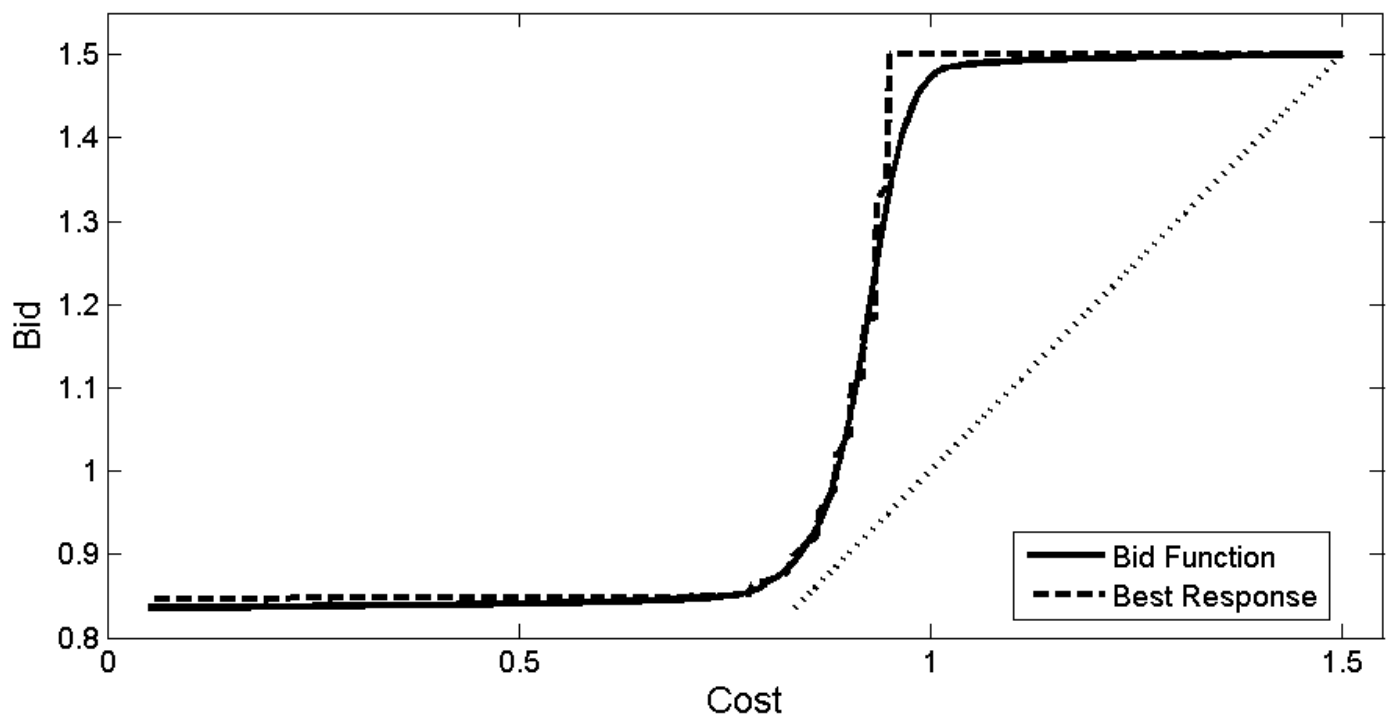

Figure 4: Computed bid function (solid) and simulated best response (dashed) for a first-price auction with parameters equal to those in the Base case in Table 4 . The dotted line is the $45^{\circ}$ line. We use 500,000 simulations to compute the best response. 


\section{TABLES}

\begin{tabular}{|c|c|c|c|c|c|c|}
\hline & Variable & Mean & Std. Dev. & $25^{\text {th }}$-tile & $50^{\text {th }}$-tile & $75^{\text {th }}$-tile \\
\hline \multirow{6}{*}{$\begin{array}{c}11 \\
0 \\
\frac{1}{0} \\
\frac{0}{0} \\
\# \\
\#\end{array}$} & Potential Bidders & 6.882 & 1.997 & 5 & 7 & 8 \\
\hline & Probability of Entry & 0.649 & 0.198 & 0.500 & 0.667 & 0.800 \\
\hline & Unemployment & 4.136 & 0.971 & 3.2 & 4.1 & 4.6 \\
\hline & Engineer Estimate & 491,120 & 543,662 & 226,338 & 320,755 & 523,139 \\
\hline & Win Bid & 451,800 & 520,945 & 207,146 & 288,317 & 471,151 \\
\hline & Relative Win Bid & 0.917 & 0.133 & 0.826 & 0.888 & 0.980 \\
\hline \multirow{6}{*}{$\begin{array}{l}\stackrel{\vec{L}}{1} \\
11 \\
\dot{20} \\
\stackrel{\dot{0}}{0} \\
\#\end{array}$} & Potential Bidders & 7.909 & 2.027 & 6 & 8 & 10 \\
\hline & Probability of Entry & 0.626 & 0.192 & 0.500 & 0.600 & 0.750 \\
\hline & Unemployment & 5.641 & 1.214 & 4.4 & 5.6 & 6.5 \\
\hline & Engineer Estimate & $1,409,158$ & $1,185,331$ & 550,537 & 967,817 & $1,977,169$ \\
\hline & Win Bid & $1,366,487$ & $1,177,504$ & 530,084 & 892,737 & $1,847,180$ \\
\hline & Relative Win Bid & 0.976 & 0.134 & 0.892 & 0.965 & 1.038 \\
\hline \multirow{6}{*}{$\begin{array}{l}0 \\
\vec{H} \\
\| \\
\dot{n} \\
\dot{0} \\
0 \\
\#\end{array}$} & Potential Bidders & 7.262 & 2.067 & 6 & 7 & 9 \\
\hline & Probability of Entry & 0.640 & 0.196 & 0.500 & 0.625 & 0.778 \\
\hline & Unemployment & 4.693 & 1.291 & 3.8 & 4.4 & 5.7 \\
\hline & Engineer Estimate & 830,971 & 949,131 & 258,485 & 446,273 & 914,745 \\
\hline & Win Bid & 790,410 & 936,689 & 240,553 & 406,155 & 887,277 \\
\hline & Relative Win Bid & 0.939 & 0.136 & 0.844 & 0.914 & 1.006 \\
\hline
\end{tabular}

Table 1: Summary statistics for various quantities, subdivided into state groups. Win Bid and Engineer Estimate are measured in dollars. Relative Win Bid is the winning bid relative to the engineer's estimate. See the text for more details of the sample.

\begin{tabular}{rcccccc}
\hline & $\beta_{0}$ & $\beta_{1}$ & $\beta_{2}$ & $\omega$ & $\underline{c}$ & $\bar{c}$ \\
\hline$\mu_{C}$ & -0.07 & -0.005 & 0 & 0.012 & -0.4 & 1.0 \\
$\sigma_{C}$ & 0.05 & 0 & 0 & 0.025 & 0.0095 & 0.995 \\
$\alpha$ & 0.5 & 0 & 0 & 0.25 & 0.1 & 0.9 \\
$K$ & 0.015 & 0 & 0 & 0.01 & $10^{-4}$ & 0.16 \\
\hline
\end{tabular}

Table 2: Importance sampling density $\tilde{\Gamma}$ for estimation. The parameter $\tilde{\Gamma}$ specifies distribution, as given by the functional form given in equation (9). 


\begin{tabular}{ccccc|cc}
\hline & $\beta_{0}$ & $\beta_{1}$ & $\beta_{2}$ & $\omega$ & OK Mean & TX Mean \\
\hline$\mu_{C}$ & -0.0868 & -0.0023 & 0.0154 & 0.0142 & $91.4 \%$ & $91.9 \%$ \\
& $(0.0324)$ & $(0.0064)$ & $(0.0132)$ & $(0.0091)$ & $(0.84 \%)$ & $(1.89 \%)$ \\
$\sigma_{C}$ & 0.0687 & & -0.0117 & 0.0304 & $6.62 \%$ & $5.33 \%$ \\
& $(0.0196)$ & & $(0.0213)$ & $(0.0132)$ & $(0.97 \%)$ & $(0.87 \%)$ \\
$\alpha$ & 0.4979 & & 0.1115 & 0.1284 & 0.4979 & 0.6094 \\
& $(0.0972)$ & & $(0.0943)$ & $(0.0764)$ & $(0.0770)$ & $(0.0752)$ \\
$K$ & -0.0018 & & 0.0105 & 0.0189 & $1.35 \%$ & $1.84 \%$ \\
& $(0.0406)$ & & $(0.0191)$ & $(0.0109)$ & $(0.26 \%)$ & $(0.28 \%)$ \\
\hline
\end{tabular}

Table 3: Parameter estimates for $\Gamma$. Standard errors are computed through a nonparametric bootstrap described in the text. Standard deviations for the means displayed in the last two columns are computed by running the procedure described in the text for each bootstrap estimate; the standard deviation of these means of expected values is then reported. All \%s are relative to the engineer's estimate. 


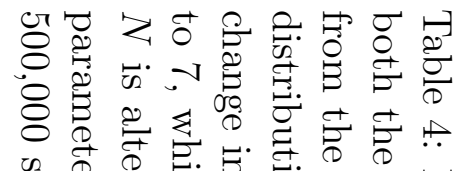

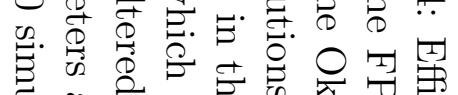

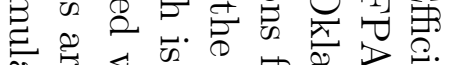

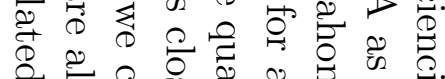
थ

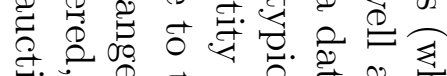
¿ ब न्य ए 东

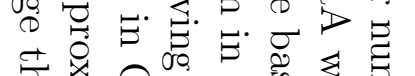

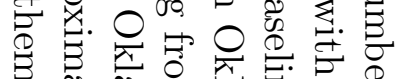

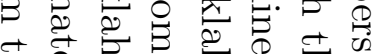
के $\begin{gathered}0 \\ 4\end{gathered}$ 它。 P

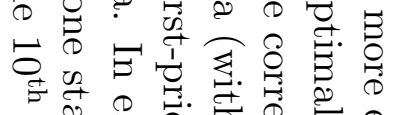
एँ

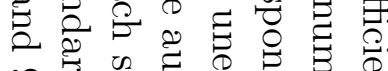
๑ जि की

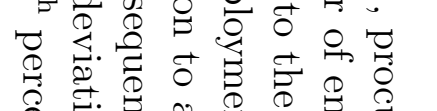

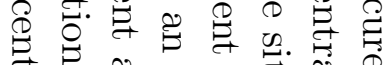

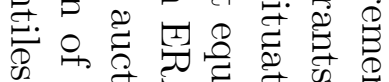
吉吉.

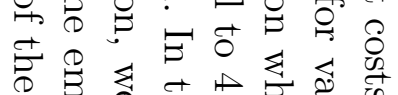

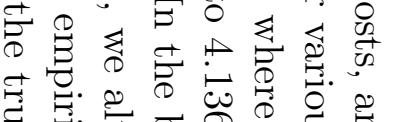
है

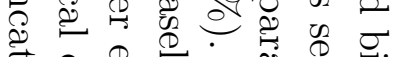

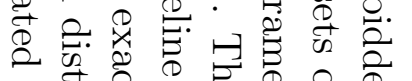
ㄹ.

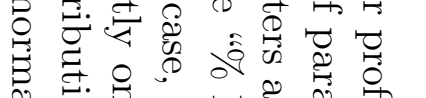

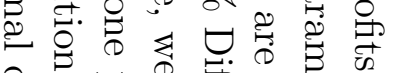

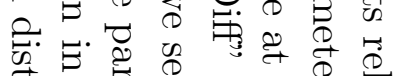

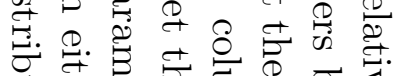

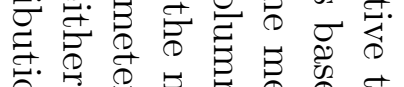
员

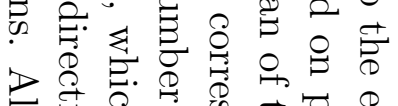

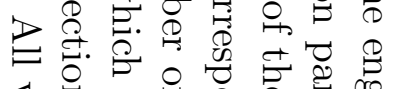

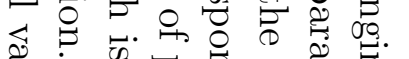

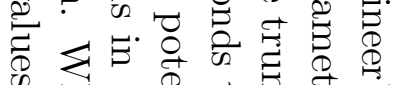

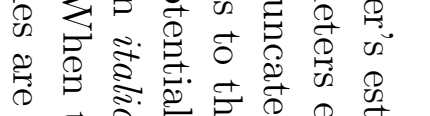

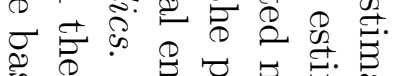

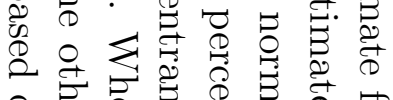

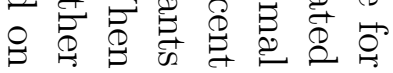

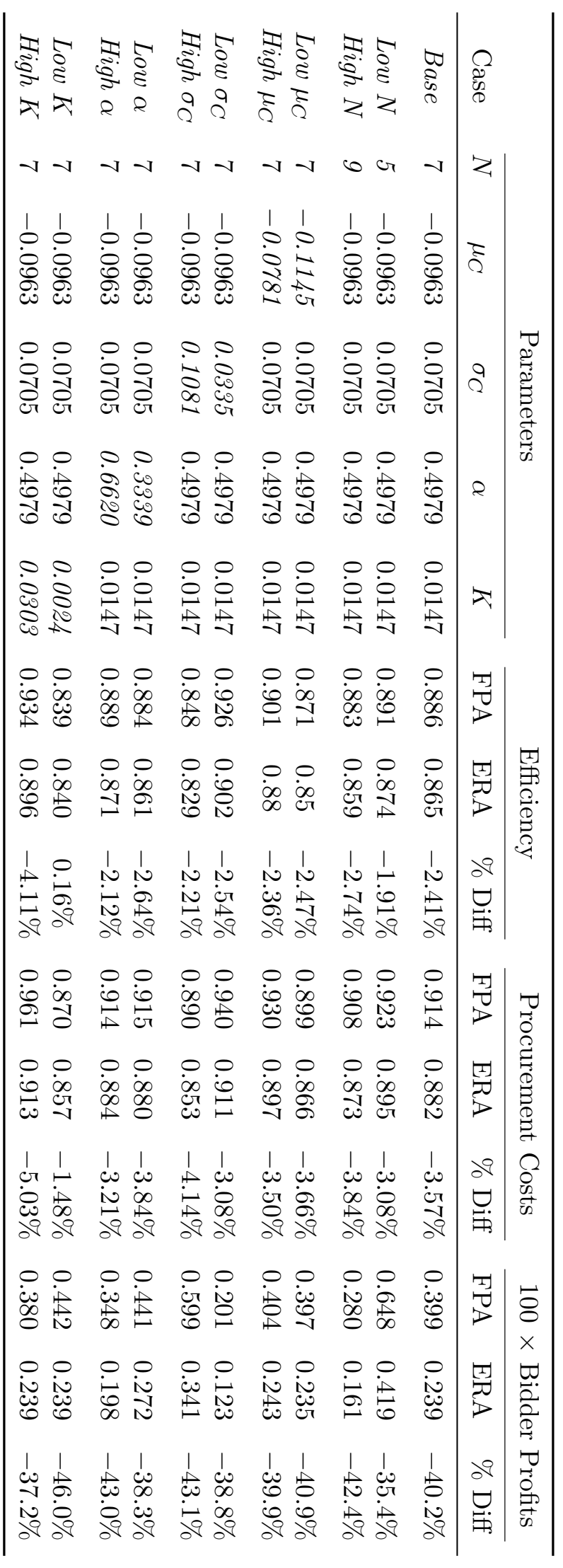




\begin{tabular}{|c|c|c|c|c|c|c|c|c|c|}
\hline \multirow[b]{2}{*}{ Case } & \multicolumn{4}{|c|}{ FPA } & \multicolumn{5}{|c|}{ ERA } \\
\hline & Comp Cost & Win Bid & Entry Prob & $s^{*}$ & Comp Cost & Win Bid & Entry Bid & $n_{\mathrm{AUC}}^{*} / N$ & $\mathbb{E}[\bar{S}]$ \\
\hline Base & 0.837 & 0.914 & 0.483 & 0.9043 & 0.835 & 0.891 & 0.0046 & $2 / 7$ & 0.8778 \\
\hline Low $N$ & 0.845 & 0.923 & 0.625 & 0.9376 & 0.844 & 0.902 & 0.0036 & $2 / 5$ & 0.9095 \\
\hline High $N$ & 0.831 & 0.908 & 0.392 & 0.8837 & 0.829 & 0.884 & 0.0051 & $2 / 9$ & 0.8587 \\
\hline Low $\mu_{C}$ & 0.821 & 0.899 & 0.485 & 0.8884 & 0.820 & 0.875 & 0.0043 & $2 / 7$ & 0.8620 \\
\hline$H i g h \mu_{C}$ & 0.852 & 0.930 & 0.481 & 0.9206 & 0.851 & 0.907 & 0.0050 & $2 / 7$ & 0.8940 \\
\hline Low $\sigma_{C}$ & 0.877 & 0.940 & 0.469 & 0.9049 & 0.873 & 0.900 & -0.0054 & $2 / 7$ & 0.8934 \\
\hline High $\sigma_{C}$ & 0.797 & 0.890 & 0.496 & 0.9068 & 0.800 & 0.882 & 0.0147 & $2 / 7$ & 0.8626 \\
\hline Low $\alpha$ & 0.835 & 0.915 & 0.473 & 0.9028 & 0.831 & 0.881 & 0.0006 & $2 / 7$ & 0.8817 \\
\hline High $\alpha$ & 0.838 & 0.914 & 0.497 & 0.9072 & 0.841 & 0.902 & 0.0088 & $2 / 7$ & 0.8717 \\
\hline Low $K$ & 0.828 & 0.870 & 0.656 & 0.9452 & 0.835 & 0.891 & 0.0169 & $2 / 7$ & 0.8778 \\
\hline High $K$ & 0.848 & 0.961 & 0.408 & 0.8874 & 0.835 & 0.891 & -0.0109 & $2 / 7$ & 0.8778 \\
\hline
\end{tabular}

Table 5: Breakdown of the expected values of major quantities in a FPA and an ERA. The cases correspond to the parameters listed in Table 4. The "Comp Cost" column reports the expected completion cost in the mechanisms, which is the winner's cost when the project is awarded to a bidder and is the outside option $c_{0}=r=1.5$ when there is no winner. The "Win Bid" column in the FPA is identically the expected procurement cost, while in the ERA, it is the winner's expected second-round bid. The "Entry Bid" column lists the expected first-round bid that the entrants have to pay in an ERA. The column $n_{\mathrm{AUC}}^{*} / N$ corresponds to the optimal number of entrants in the ERA. Finally, $\mathbb{E}[\bar{S}]$ lists the expected value of the $\left(n_{\mathrm{AUC}}^{*}+1\right)^{\mathrm{st}}$ lowest signal. 


\begin{tabular}{|c|c|c|c|c|c|}
\hline \multirow[b]{2}{*}{ Parameter } & \multirow[b]{2}{*}{ Variable } & \multirow[b]{2}{*}{ True Value } & \multicolumn{3}{|c|}{ Experiment } \\
\hline & & & $\mathrm{A}$ & B & $\mathrm{C}$ \\
\hline \multirow[t]{3}{*}{ Location Parameter $\left(\mu_{C}\right)$} & Constant & 0 & $\begin{array}{l}0.0000 \\
(0.0100)\end{array}$ & $\begin{array}{l}0.0015 \\
(0.0186)\end{array}$ & $\begin{array}{c}-0.0043 \\
(0.0170)\end{array}$ \\
\hline & $x_{a}$ & 0.65 & $\begin{array}{l}0.6507 \\
(0.0174)\end{array}$ & $\begin{array}{l}0.6533 \\
(0.0282)\end{array}$ & $\begin{array}{l}0.6602 \\
(0.0247)\end{array}$ \\
\hline & $\omega$ & 0.05 & $\begin{array}{l}0.0488 \\
(0.0044)\end{array}$ & $\begin{array}{r}0.0575 \\
(0.0073) \\
\end{array}$ & $\begin{array}{l}0.0504 \\
(0.0059) \\
\end{array}$ \\
\hline \multirow[t]{3}{*}{ Scale Parameter $\left(\sigma_{C}\right)$} & Constant & 0.05 & $\begin{array}{l}0.0494 \\
(0.0074)\end{array}$ & $\begin{array}{l}0.0463 \\
(0.0152)\end{array}$ & $\begin{array}{l}0.0416 \\
(0.0126)\end{array}$ \\
\hline & $x_{a}$ & 0 & $\begin{array}{c}-0.0002 \\
(0.0113)\end{array}$ & $\begin{array}{l}0.0085 \\
(0.0204)\end{array}$ & $\begin{array}{l}0.0195 \\
(0.0194)\end{array}$ \\
\hline & $\omega$ & 0.02 & $\begin{array}{l}0.0192 \\
(0.0035)\end{array}$ & $\begin{array}{l}0.0295 \\
(0.0094)\end{array}$ & $\begin{array}{l}0.0225 \\
(0.0077)\end{array}$ \\
\hline \multirow[t]{3}{*}{ Degree of Selection $(\alpha)$} & Constant & 0.5 & $\begin{array}{l}0.4994 \\
(0.0205)\end{array}$ & $\begin{array}{l}0.4985 \\
(0.0354)\end{array}$ & $\begin{array}{l}0.4984 \\
(0.0231)\end{array}$ \\
\hline & $x_{a}$ & 0 & $\begin{array}{l}0.0000 \\
(0.0308)\end{array}$ & $\begin{array}{c}-0.0102 \\
(0.0607)\end{array}$ & $\begin{array}{c}-0.0130 \\
(0.0571)\end{array}$ \\
\hline & $\omega$ & 0.05 & $\begin{array}{l}0.0479 \\
(0.0078)\end{array}$ & $\begin{array}{l}0.0803 \\
(0.0190)\end{array}$ & $\begin{array}{l}0.0561 \\
(0.0164)\end{array}$ \\
\hline \multirow[t]{3}{*}{ Entry Cost $(K)$} & Constant & 0.04 & $\begin{array}{l}0.0388 \\
(0.0047)\end{array}$ & $\begin{array}{l}0.0361 \\
(0.0118)\end{array}$ & $\begin{array}{l}0.0387 \\
(0.0085)\end{array}$ \\
\hline & $x_{a}$ & 0.05 & $\begin{array}{l}0.0516 \\
(0.0074)\end{array}$ & $\begin{array}{l}0.0559 \\
(0.0173)\end{array}$ & $\begin{array}{l}0.0529 \\
(0.0121)\end{array}$ \\
\hline & $\omega$ & 0.015 & $\begin{array}{l}0.0143 \\
(0.0025)\end{array}$ & $\begin{array}{l}0.0209 \\
(0.0063)\end{array}$ & $\begin{array}{l}0.0145 \\
(0.0038)\end{array}$ \\
\hline
\end{tabular}

Table 6: Monte Carlo simulations, with standard deviations of the estimates in parentheses. All Monte Carlo runs use 500 auctions as data. Column A corresponds to the infeasible estimator that uses the true distribution as the importance sampling distribution, with 2,500 simulation auctions per run. Column B corresponds to using a wider distribution as the sampling distribution, with 2,500 simulation auctions per run. Column $\mathrm{C}$ corresponds to using the wider distribution, with 15,000 simulation auctions per run. Note that 2,500 simulations per run corresponds to using 5 simulations per auction in the data (and 15,000 simulations per run correspond to using 30 simulations per auction) since simulations are drawn without replacement from the pool of all simulations computed. 